\title{
Natur als sakraler Raum in der minoischen Kultur
}

\section{Diamantis Panagiotopoulos}

„... keine kulturanthropologische Forschung scheint bei der Bestimmung von ,Religion' auf die Scheidung in einen profanen und einen sakralen Bereich verzichten zu können." "

\section{Vorbemerkungizu den methodischen Zwängen des historischen Kontextes}

Unsere Vorstellung von der Andersartigkeit der minoischen Kultur ist sowohl auf konkreter Evidenz als auch auf deren Fehlen begründet: Nicht nur die vorhandenen archäologischen und ikonographischen Zeugnisse, sondern auch eine Reihe von kulturellen Erscheinungen, die trotz intensiver Forschungsaktivität nicht dokumentiert werden konnten, haben die wissenschaftliche Erkenntnis einer bronzezeitlichen Gesellschaft mit einer ganz besonderen Mentalität genährt. $\mathrm{Zu}$ den auffälligsten Lücken in der archäologischen Überlieferung zählt das Fehlen von Tempeln, jenem monumentalen Aspekt religiöser Praxis, der im Kontext der orientalischen Kulturen das Erscheinungsbild einer Stadt oder eines Heiligtums entscheidend prägte. Der künstlich erschaffene sakrale Raum hat in der minoischen Kultur tatsächlich nur spärliche archäologische Spuren hinterlassen. Sieht man von einzelnen ,Kultbauten' ab, die als architektonisches Konzept sehr bescheiden und keineswegs distinktiv sind, ${ }^{2}$ gibt es eigentlich kaum freistehende Tempel.

Folgende Abkürzungen werden verwendet:

Eliade, Das Heilige

Bollnow, Mensch und Raum

Fickeler, Religionsgeographie
M. Eliade, Das Heilige und das Profane. Vom Wesen des Religiösen (1990)

O.F. Bollnow, Mensch und Raum (19947)

P. Fickeler, "Grundfragen der Religionsgeographie", in: M. Schwind (Hg.), Religionsgeographie (1975) 48-99 (Nachdruck der in Erdkunde I [1947] I 2 I-I44 veröffentlichen Originalfassung)

I Handbuch religionswissenschaftlicher Grundbegriffe IV (1998) 398 s.v. „Raum“ (R. Gehlen).

2 Der wichtigste dieser aus architektonischer Sicht eher unscheinbaren sakralen Räume ist der sogenannte Tempel von Anemospilia, dessen freigelegter Teil dem Grundrissplan eines 
Minoische Heiligtümer sind uns etwas besser bekannt, doch beschränkt sich hier die architektonische oder künstlerische Formung der sakralen Sphäre auf umfriedete Kultbezirke oder die Konstruktion von Gebäuden, die verschiedene Zwecke erfüllten, nur nicht den eines sakralen Raumes für die Austragung von kultischen Handlungen. ${ }^{3}$ Kleine Schreine oder Kapellen, die in größere Baukomplexe eingebettet sind, sowie Kulträume in elitären Häusern, Villen oder kleinen Dörfern sind weniger mit gemeinschaftlicher kultischer Aktivität und kollektiver religiöser Erfahrung, als vielmehr mit den Belangen einer privaten Pietät zu verknüpfen. ${ }^{4}$ In all diesen Fällen lässt sich kein religiöses Konzept erkennen, das in architektonischen Formen Ausdruck gefunden hat. Jeder Versuch, dieser recht bescheidenen und rein zweckmäßigen Architektur symbolische Bedeutung abzugewinnen, wäre zwecklos gewesen.' Das rätselhafte Fehlen einer ausgeprägten, symbolisch beladenen sakralen Baukunst wird umso problematischer, wenn man an die sehr aufwendige architektonische Ummantelung des elitären Lebensraumes denkt, die sich nicht nur im minoischen Palast, sondern auch in den anspruchsvollen Innenräumen von Villen und vornehmen Privathäusern manifestiert. Bei diesen Bauten kann man die klare Umsetzung eines idealtypischen bautechnischen Konzeptes erkennen, das man im sakralen Kontext vermisst. Hat sich tatsächlich in der minoischen Kultur keine sakrale Architektur entfaltet, wie wir sie aus anderen Kulturregionen des östlichen Mittelmeers kennen? Trotz des eindeutig negativen archäologischen Befundes ist bei der Beantwortung dieser Frage Vorsicht geboten. Was uns zur Vorsicht mahnt, sind verschiedene Architekturdarstellungen, die als Fassaden

Magazintraktes ähnelt und z.T. auch tatsächlich diese Funktion erfüllte, s. hierzu Y. Sakellarakis - E. Sakellarakis, Archanes. Minoan Crete in a New Light. (1997) 269-3 I I.

3 A. Lebessi - P. Muhly, „Aspects of Minoan Cult. Sacred Enclosures. The Evidence from the Syme Sanctuary (Crete)“, Archäologischer Anzeiger 1990, 31 5-336; A. Lebessi, „A Minoan Architectural Model from the Syme Sanctuary, Crete“, Mitteilungen des Deutschen Archäologischen Instituts, Athenische Abteilung. 1 I 7 (2002) I-19. Zu den minoischen Okkupationsphasen dieses Heiligtums liegen uns sonst nur Vorberichte vor, s. A. Lebessi u. a., "The Runner's Ring, a Minoan Athlete's Dedication at the Syme Sanctuary, Crete", Mitteilungen des Deutschen Archäologischen Instituts, Athenische Abteilung. I 9 (2004) I Anm. I-2. Vgl. ferner die minoischen Höhenheiligtümer, auf die im Folgenden näher eingegangen werden soll.

4 G.C. Gesell, Town, Palace, and House Cult in Minoan Crete. Studies in Mediterranean Archaeology 67 (1985); L. A. Hitchcock, Minoan Architecture. A Contextual Analysis (2000) 102-109. Problematisch bleibt die Deutung von Kulträumen in den Palästen von Phaistos und Malia, die nur von außen zugänglich sind und folglich einen öffentlicheren Charakter gehabt zu haben scheinen, s. hierzu Hitchcock, a. O. 72-73.

5 Zur Bedeutung der Architekturcodes (Absonderung, optische Qualifizierung, Monumentalität), die den Tempel und seinen Bezirk als sakrale Sphäre in seiner praktischen und symbolischen Funktion vom übrigen Stadtbild abheben, s. z. B. B. Pongratz-Leisten, Ina sulmi irub. Die kulturtopographische und ideologische Programmatik der akitu-Prozession in Babylonien und Assyrien. Bagdader Forschungen 16 (1994) 20. 
kultischer Gebäude gedeutet werden. ${ }^{6}$ Eine Auseinandersetzung mit den Debatten, die diese ikonographischen Zeugnisse ausgelöst haben, würde den Rahmen des vorliegenden Beitrags sprengen. Halten wir hier fest, dass die Fokussierung auf die Natur als sakralen Raum im minoischen Kreta zum jetzigen Kenntnisstand alternativlos ist, da es keine sichere Grundlage für die Untersuchung einer sakralen Architektur gibt. Im Versuch, aus dieser forschungsgeschichtlich bedingten Not eine Tugend zu machen, konzentrieren sich folgende Überlegungen auf den tatsächlich besonderen religiösen Umgang der Minoer mit ihrer natürlichen Umgebung.

\section{Sakraler Raum: Versuch einer Definition}

Fangen wir mit einem Axiom an, das eigentlich den Charakter einer Selbstverständlichkeit hat: Jede vormoderne Gesellschaft hat ihre Natur in irgendeiner Weise sakralisiert. Die Vorstellung vom sakralen Charakter der Natur, von der Heiligkeit der Mutter Erde, mag sicherlich in den verschiedenen vormodernen Kulturen in unterschiedlicher Intensität und Ausprägung auftreten, ist allerdings überall präsent.? Insbesondere die religiösen Vorstellungen der Naturvölker sind durch einen starken seelischen Bezug der Menschen zu ihrer Umwelt geprägt. Dieser Grundgedanke über die sakralisierte Natur als Kern des religiösen Kosmos, den wir auch im Kontext der minoischen Kultur voraussetzten dürfen, kann uns eigentlich wegen seiner Allgemeinheit nicht wesentlich weiter bringen, wenn es

6 Es handelt sich in erster Linie um Darstellungen des sogenannten dreiteiligen Säulenheiligtums, s. Th. Nörling, Altägäische Architekturbilder. Archaeologica Heidelbergensia 2 (1995) 54-6o; Hitchcock, Minoan Architecture, a. O. (Anm. 4) I02-104. Zu einer profanen Deutung dieses Motivs als ,Piktogramm' des Palastes s. K. Krattenmaker, "Palace, Peak and Sceptre: The Iconography of Legitimacy", in: P. Rehak (Hg.), The Role of the Ruler in the Prehistoric Aegean. Proceedings of a Panel Discussion Presented at the Annual Meeting of the Archaeological Institute of America, New Orleans, Louisiana, 28 December 1992, with Additions. Aegaeum I I (1995) 49-58. Zu alternativen Interpretationen des Gebäudes auf dem reliefierten Rhyton aus Kato Zakros, das als eines der bekanntesten Beispiele des ,dreiteiligen Säulenheiligtums' gilt, s. ferner E. E. Bloedow, „The Sanctuary Rhyton from

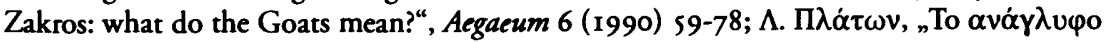

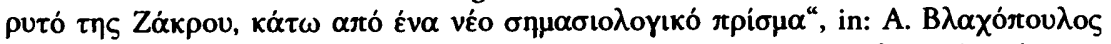

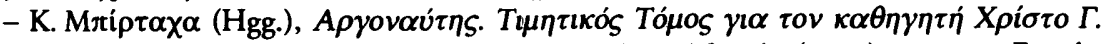

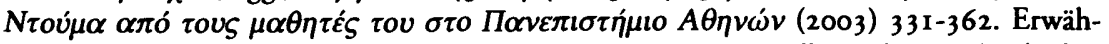
nenswert ist schließlich in diesem Zusammenhang ein Tonmodell aus dem Höhenheiligtum von Petsophas, das ein sakrales (?) Gebäude in Kulthörnerform darstellt, s. N. Marinatos, Ritual, Image, and Symbol (1993) I 2 I Abb. 86.

Eliade, Das Heilige 103. 
uns darum geht, das Verhältnis zwischen Mensch und Naturraum in einem spezifischen kulturellen Kontext zu ergründen.

Eine weitaus größere Bedeutung hat in unserem Zusammenhang die Erkenntnis, dass der erlebte Raum und dadurch auch die Natur nicht homogen sind. Die religiöse Bedeutungsgliederung des Raumes hat E. Cassirer in seiner „Philosophie der symbolischen Formen“ explizit gemacht: „Die Heiligung beginnt damit, dass aus dem Ganzen des Raumes ein bestimmtes Gebiet herausgelöst, von anderen Gebieten unterschieden und gewissermaßen religiös umfriedet und umhegt wird." ${ }^{\text {" }}$ Dieses dualistische Konzept der Strukturierung des erlebten Raumes fand später durch M. Eliade in seiner bahnbrechenden Abhandlung „Das Heilige und das Profane“ eine eingehende Behandlung. Das erste Kapitel von Eliades nunmehr klassischem Text beginnt mit folgenden Worten, die uns eine sehr einfache aber auch luzide Definition der Spaltung des vom Menschen erlebten Raumes in zwei Sphären bieten: „Für den religiösen Menschen ist der Raum nicht homogen; er weist Brüche und Risse auf: er enthält Teile, die von den übrigen qualitativ verschieden sind [...]. Es gibt also einen heiligen, d.h. ,starken', bedeutungsvollen Raum, und es gibt andere Räume, die nicht heilig und folglich ohne Struktur und Festigkeit, in einem Wort amorph sind. Mehr noch: diese Inhomogenität des Raumes erlebt der religiöse Mensch als einen Gegensatz zwischen dem heiligen, d.h. dem allein wirklichen, wirklich existierenden Raum und allem übrigen, was ihn als formlose Weite umgibt."9 Wir dürfen also davon ausgehen, dass jede vormoderne Gesellschaft ihren Naturraum in einen sakralen und profanen Raum strukturiert hat. Der sakrale Raum besteht aus religiösen Bedeutungsorten als Formen ritueller Bündelung und Verdichtung, Orte, die einen Bruch in der Homogenität des Raums darstellen..$^{10}$ Eine sakrale Landschaft ist allerdings weit mehr als die Summe der

8 E. Cassirer, Philosophie der symbolischen Formen 23 (1958) I 23 (zitiert in Bollnow, Mensch und Raum 1 43-144). Zum Begriffspaar Heiliges und Profanes als grundlegendem Unterscheidungsmerkmal religiösen Denkens s. bereits E. Durkheim, Die elementaren Formen des religiösen Lebens ( 198 I) 62-68.

9 Eliade, Das Heilige 23. Zu profaner und sakraler Raumqualität s. auch A. Schart, „Die Entgrenzung des heiligen Raumes. Tempelkonzept und Tempelkritik in der biblischen Tradition“, Pastoraltheologie 86 (1997) 348: „Der Raum gewinnt seine Gestalt durch die dynamischen Potenzen, die ihn erfüllen. Eine ganz wesentliche Raumstruktur wird hervorgerufen durch die Anwesenheit des Heiligen. Die Manifestation des Heiligen schafft eine heilige Stätte, die sich scharf aus der profanen Sphäre ausgrenzt. Sie stellt den absoluten Fixpunkt dar, der zielgerichteter menschlicher Bewegung die Orientierung ermöglicht.“

Io Eliade, Das Heilige 36. Zu diesem dualistischen Raumschema und der daraus resultierenden Strukturierung von Alltags- und Sakraltopographien s. ferner B. Hauser-Schäublin, „Raum, Ritual und Gesellschaft. Religiöse Zentren und sozio-religiöse Verdichtungen im Ritual“", in: Dies. - M. Dickhardt (Hgg.), Kulturelle Räume - räumliche Kultur. Zur Neubestimmung des Verhältnisses zweier fundamentaler Kategorien menschlicher Praxis. Göttinger Studien zur Ethnologie ro (2003) bes. 45-47. Nach Hauser-Schäublin sind sakrale Land- 
aus ihrer profanen Umgebung herausgehobenen heiligen Stätten. Durch heilige Prozessionen und Pilgerreisen, die als ,Kraftlinien' die heiligen Orte miteinander und mit den Besiedlungszentren verbinden, werden sakrale Plätze in ein kultreligiöses ,Kraftfeld 'verwoben. ${ }^{\text {II }}$ Durch ihr zeremonielles Durchschreiten gewinnt die sakrale Landschaft Textur. Neben ihrer Ausgrenzung aus dem übrigen amorphen Raum zeichnen sich heilige Räume besonders durch ihren Schwellencharakter aus. Sie sind liminale Orte, die nicht nur den Göttern vorbehalten sind, sondern ihre primäre Funktion als Zwischenräume, Schnittpunkte und Schwellen zwischen den beiden Welten erfüllen: zwischen den Menschen auf dieser Erde und den Göttern, die immer in einer ganz anderen Sphäre angesiedelt sind.

Wie kann man nun erklären, dass an bestimmten Orten eine besondere, religiöse Bedeutung haftet? Was macht aus einem Ort einen kraftgeladenen, bedeutungsvollen Raum? Woraus schöpfen sakrale Stätten ihre symbolische Valenz? Für die Zweiteilung des erlebten Raumes in eine sakrale und eine profane Sphäre durch die Überhöhung von bestimmten Stätten zu heiligen Plätzen bestehen grundsätzlich drei Optionen: Die Sakralität eines Ortes kann a) von der Landschaftsphysiognomie, in anderen Worten von der Lage oder der besonderen atmosphärischen Wirkung eines Ortes, abhängen, ${ }^{12}$ b) mit dem Wirken einer Person oder mit einer Begebenheit verknüpft $\operatorname{sein}^{13}$ oder c) durch Rituale pauschal bzw. repetitiv erzeugt werden. ${ }^{14}$ Sakralität erweist sich hier verschiedentlich als Resultat der kognitiven Wahrnehmung (a), der Erinnerung (b) oder der Ritualaktion (c). ${ }^{\text {Is }}$ Im Fall des

schaften als ,leere Stellen' der Alltagstopographie zu begreifen, die menschlichem Handeln auf permanenter oder temporärer Basis nicht zugänglich sind. Zu heiligen Orten als herausgehobenen Stätten s. ferner Bollnow, Mensch und Raum, 143.

I I s. Fickeler, Religionsgeographie 75; ferner R. Gehlen, Welt und Ordnung. Zur soziokulturellen Dimension von Raum in frühen Gesellschaften (1995) I 84-187.

I s. auch Fickeler, Religionsgeographie 8o. Zu Naturheiligtümern s. ferner G. van der Leeuw, Phänomenologie der Religion (1956) 445-447.

I3 Diese beiden ersten Parameter der Sakralität können als ,naturhaft-magische' und ,geschichtlich-religiöse Heiligkeit' bezeichnet werden, s. Fickeler, Religionsgeographie 55. Zu einer stark anthropo- bzw. theozentrisch geprägten Definition des sakralen Ortes, die nicht vom Potential der Orte selbst ausgeht, s. S. Japhet, „Some Biblical Concepts of Sacred Place", in: B.Z. Kedar - R.J. Zwi Werblowsky (Hgg.), Sacred Space. Shrine, City, Land (1998) $57-70$.

I4 Ein gutes Beispiel für diese Option bietet die christliche Liturgiefeier, die nicht auf einen sakralen Raum angewiesen ist, sondern ihn um sich schafft. Zu religiösen Ritualen als Auslösern eines eigenständigen Raumverständnisses, s. R. Volp, „Das offene Labyrinth. Über den Wechselbezug zwischen Raum- und Ritualbewusstsein“, in: Th. Nißlmüller - R. Volp (Hgg.), Raum als Zeichen. Wabrnehmung und Erkenntnis von Räumlichkei. Ästhetik - Theologie - Liturgik I (1998) 25.

I5 s. hierzu auch H. Cancik, „Rome as Sacred Landscape. Varro and the End of Republican Religion in Rome“, Visible Religion 4-5 (1985-1986) 253: „Sacred landscape is a constellation of natural phenomena constituted as a meaningful system by means of artificial and 
minoischen Kreta könnte man mit guten Gründen für erstere Option plädieren und die sakrale Potenz der minoischen heiligen Stätten mit der besonderen physiognomischen Qualität eines Ortes in Beziehung setzen. Was uns dazu veranlasst, ist vordergründig der besondere Charakter der kretischen Landschaft, auf den im Folgenden näher eingegangen wird. Gemäß dieser Deutung wären die minoischen Rituale keine Mittel zur Erzeugung von Sakralität, sondern schlicht eine Bestätigung dieser Sakralität. Dabei lässt sich natürlich keineswegs ausschließen, dass auch in der minoischen Gesellschaft sakrale Orte entweder als Schauplätze einer mythischen Episode begriffen oder durch Rituale an einer beliebigen Stelle des homogenen Raumes erzeugt werden konnten. Diese beiden Optionen der Sakralisierung lassen sich allerdings ohne schriftliche Quellen kaum belegen.

\section{Die kretische Landschaft: Diversität der Natur und ihr sakrales Potential}

Verlassen wir diese theoretische Ebene und versuchen wir in einem ersten Anlauf zu unserer Problematik einen kursorischen Blick auf die Materialität der kretischen Landschaft zu werfen. ${ }^{16}$ Welche sind die natürlichen Eigenschaften dieses Naturraumes und wie helfen sie uns, das Spezifikum der minoischen naturnahen Religion greifbar zu machen? Die kretische Landschaft zeichnet sich besonders durch Vielfalt und einen menschlichen Maßstab der Dinge aus. Vielfalt erkennt man an den stets abwechselnden naturräumlichen Elementen, an den zahlreichen Bezugspunkten des Blickes, an den bewegten und immer ungeraden Linien der Hügel und Berge, die das Erscheinungsbild der Insel dominieren (Abb. I). Die Landschaftsformen haben durch ihre Kleinkammerigkeit einen menschlichen Maßstab, da

religious signs, by telling names or etiological stories fixed to certain places, and by rituals which actualize the space." Pongratz-Leisten unterscheidet in diesem Zusammenhang zwischen zwei Formen der Sakralisierung eines Ortes: a) eine, Mythologisierung', die sich auf die Ebene der mythischen Erzählungen bezieht, und b) eine ,Ritualisierung', die sich durch symbolische Handlungen vollzieht, s. Pongratz-Leisten, Ina šulmi irub, a. O. (Anm. 5) is16. Diese begriffliche Unterscheidung zeigt eine spürbare Referenz zur Zweiteilung der numinosen Orte K. Hübners in solche, die in den profanen Raum eingebettet sind, und solche, die für die Menschen unzugänglich sind, s. K. Hübner, Die Wabrbeit des Mythos (1985) $165 \mathrm{f}$. Man darf hier vermuten, dass Erstere in der Regel ritualisiert, Letztere hingegen mythologisiert wurden.

16 Hier muss einleitend angemerkt werden, dass im Fall Kretas, einer dramatischen Insellandschaft voller Kontraste und enormer Höhenunterschiede, die Diskrepanz zwischen dem abstrakten geometrischen Raum, den unsere Karten wiedergeben, und dem hodologischen Raum, nämlich dem erlebten, durch Wege erschlossenen geographischen Raum, gravierend ist. Täler, Schluchten, kleine Anhöhen, Hügel und Bergketten machen das kretische Land weitaus größer, vielfältiger und letztendlich ganz andersartig als das durch Karten vermittelte Bild. Zum hodologischen Raum s. Bollnow, Mensch und Raum 191-202. 
jede Region klein, in sich geschlossen und überschaubar ist. Ihre Grenzen können schnell, höchstens im Fußmarsch eines Tages erreicht werden. Der Mensch lebt im Zentrum einer kleinen geographischen Einheit, die er immer mit seinem Blick erfassen bzw. mit wenig Mühe begehen kann, und fühlt sich daher als $\mathrm{Maß}$ aller Dinge, was in ihm das Gefühl eines sehr harmonischen Verhältnisses zur Natur hervorruft. Dieses immer neu aufgefaltete Land mit seinen abrupten oder fließenden Über-

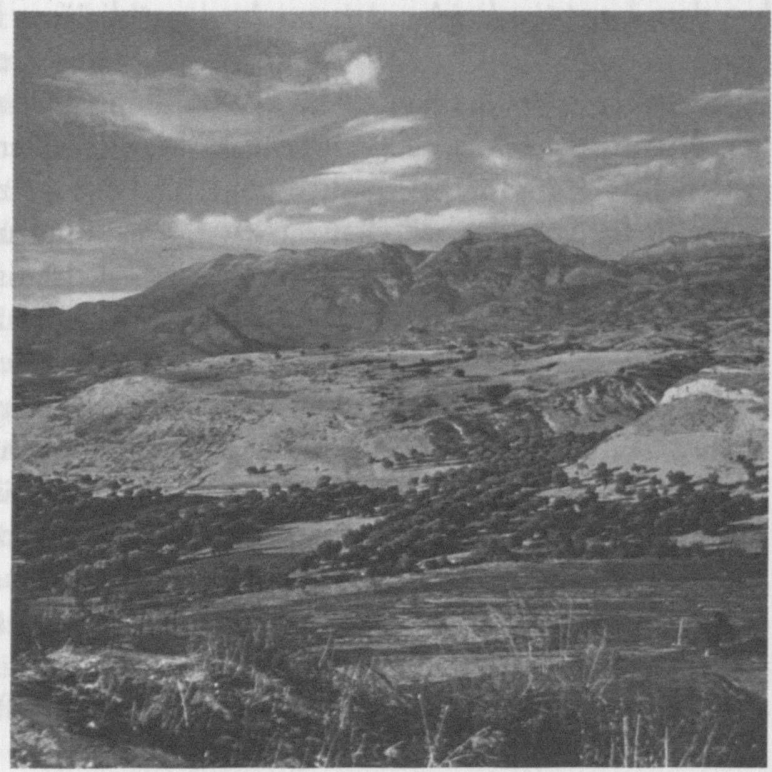

Abb. I: Kretische Landschaft. Blick vom minoischen Palast von Phaistos auf das Ida-Bergmassiv. gängen besitzt eine Fülle von besonderen Orten, die über Jahrhunderte oder gar Jahrtausende hinweg als Fixpunkte religiöser Erfahrungen fungierten. ${ }^{17}$ Kleine abgelegene Buchten, markante Erhebungen in den zahlreichen Ebenen und Tälern der Insel, aber vor allem besondere Orte in der Abgeschiedenheit der Berge wie Felsen, Steine, Karstdepressionen, Poljen, Hochebenen, Höhlen, Quellen und Bäume entfalten eine ganz besondere atmosphärische Wirkung. ${ }^{18}$ Es grenzt sicherlich an Banalität festzustel-

I7 Die Sakralität, die einem besonderen Ort von Natur aus anhaftet, seine ,naturhaftmagische' Heiligkeit (s.o. Anm. 13), kann man als eine zeit- und religionsunabhängige Konstante betrachten, s. Bollnow, Mensch und Raum I43. Zu dieser ,Beharrungsregel sakraler Räume s. Fickeler, Religionsgeographie 55.

I 8 Zum Naturraum als ästhetischer Kategorie s. G. Böhme, Atmosphäre. Essays zur neuen Ästhetik (1995) 177-190. Landschaftsformationen, die von der Phantasie der Menschen belebt und mit dem Numinosen in Verbindung gebracht werden, bilden seit jeher die religiösen Verankerungsorte der erfahrbaren und konstruierten Welt einer gesellschaftlichen Gruppe. Zur Landschaft und Religion s. u. a. van der Leeuw, Phänomenologie, a. O. (Anm. 12) 445; G. Rinschede, Religionsgeographie (1999) 83-89; ferner A. Michaels, „The Sacredness of (Himalayan) Landscapes“, in: N. Gutschow u. a. (Hgg.), Sacred Landscape of the Himalaya. Österreichische Akademie der Wissenschaften, Phil.-Hist. Klasse. Denkschriften 308 (2003) I3I8. Als Bestandteil der imaginären religiösen Topographie des minoischen Kreta darf auch das Meer betrachtet werden. Auffallend ist in diesem Zusammenhang die Dominanz von 
len, dass der genius loci Ausgangspunkt einer religiösen Empfindung und damit der Sakralisierung eines Ortes sein kann. In einer in rezenten Studien viel zitierten Passage aus seinen Briefen hat Seneca den numinosen Charakter und die Atmosphäre von Naturorten sehr treffend erfasst: „Wenn du einem Haine nahest, der durch zahlreiche alte und ungewöhnlich hohe Bäume ausgezeichnet ist und in dem der Schatten der einander bedeckenden Zweige den Eindruck des Himmelsdaches hervorruft: die schlanke Höhe der Bäume, das geheimnisvolle Dunkel des Orts, die Bewunderung des so augenscheinlich dichten und durch nichts unterbrochenen Schattens ruft in dir den Glauben an eine Gottheit wach. Und wo eine tiefe Grotte sich unter überhängenden Felsen in den Berg hineinzieht, nicht von Menschen gemacht, sondern durch Naturkräfte so weit ausgehöhlt, wird deine Seele von der Ahnung des Göttlichen durchlebt werden. Großer Flüsse Ursprung verehren wir. Wo irgendwo unvermittelt ein gewaltiger Strom hervortritt, stehen Altäre. Verehrungswürdig sind warme Quellen, und manchen Seen hat schattiges Dunkel oder unergründliche Tiefe Heiligkeit verliehen. ${ }^{\text {"19 }}$ Die kretische Landschaft besitzt eine Fülle von solchen Orten, die einen idealen Schauplatz für die Begegnung mit dem Numinosen bieten, und hat somit ein hohes sakrales Potential. ${ }^{20}$

Trotz der Gefahr, in einen geodeterministischen Ansatz zu verfallen, dürfte man eine Gegenüberstellung zwischen der kretischen und der ägyptischen Landschaft bezüglich ihres potentiellen Einflusses auf die religiöse Praxis wagen. Der Vielfalt und Abwechselung, den bewegenden Umrissen, den zahlreichen und klar abgegrenzten besonderen Orten Kretas steht die Einförmigkeit der Nillandschaft mit den sich ins Unendliche fortsetzenden horizontalen Achsen der Landschaftselemente gegenüber: der Fluss, der schmale fruchtbare Landstrich an seinen Ufern und die Wüste. Der Nil, die Lebensquelle Ägyptens, fließt langsam, größtenteils geradlinig, in einer festgesetzten, unveränderten Richtung und strahlt keine Dyna-

Meeresmotiven im Keramikdekor der letzten Phase der kretischen Neupalastzeit. Diese Meeresornamentik könnte z.T. einen sakralen Hintergrund gehabt haben, s. W. Müller, Kretische Tongefäße mit Meeresdekor. Entwicklung und Stellung innerhalb der. Feinen Keramik von Spätminoisch I B auf Kreta. Archäologische Forschungen 19 (1997) 23-24. 320-322.

19 Seneca, epistulae 4, 12 (4I), 3 (Übers. O. Kern; zitiert in van der Leeuw, Phänomenologie a.O. [Anm. 12] 447). G. Böhme spricht in Bezug auf solche atmosphärischen Orte über „unbestimmt in die Weite ergossene Gefühle, die als ergreifende Mächte erfahren werden“, s. G. Böhme, „Atmosphären kirchlicher Räume“, Kunst und Kirche (1998/2) roo. Dass Raumwahrnehmung keine bloß rezeptive, sondern eine schöpferische Aktivität ist, betont s. D. Ipsen, Ort und Landschaft (2006) 19. Von einem wahrnehmungspsychologischen Standpunkt aus könnte man folglich bestimmte Kultpraktiken, die sich in Naturräumen vollziehen, als eine Art ritueller Bändigung dieses schöpferischen Elements des Erlebens und Deutens der natürlichen Umgebung betrachten.

20 Die Beziehung zwischen naturräumlicher Vielfalt und hoher Anzahl von potentiellen sakralen Orten machen auch P. Horden - N. Purcell, The Corrupting, Sea. A Study of Mediterranean History (2000) 408-4I I explizit. 
mik, sondern Beständigkeit aus. In diesem sehr stark homogenen Naturraum sind besondere Orte, die sich durch einen wirkungsvollen genius loci von der Einförmigkeit ihrer Umgebung auszeichnen, rar. Es wäre nicht ganz abwegig zu vermuten, dass man in einer solchen natürlichen Umgebung den sakralen Raum in den meisten Fällen künstlich erschaffen, d. h. architektonisch gestalten musste, damit er als ein bedeutungsvoller Ort, als Haus Gottes oder als Schnittstelle zwischen Gott und Mensch wahrgenommen werden konnte. Im geographischen Kontext der minoischen Kultur scheint auf der anderen Seite ein solcher architektonischer Eingriff in das natürliche Milieu meist überflüssig gewesen zu sein. Vielleicht hatten die Minoer kein besonders ausgeprägtes Bedürfnis, einen Tempel als Haus Gottes und Kristallisationspunkt religiöser Erfahrung zu bauen, da ihnen die Natur so viele Orte mit enormer suggestiver Kraft bot. Diese Gegenüberstellung mag vielleicht zu vereinfacht klingen, doch kann es keinen Zweifel daran geben, dass die natürliche Umwelt einen gewissen Einfluss auf religiöse Vorstellungen ausübt.

Damit berühren wir eine Frage, die noch im Mittelpunkt aktueller theologischer und religionsgeschichtlicher Debatten steht. ${ }^{2 I}$ Der dialektische Prozess zwischen einem religiösen Menschen und der Natur ist dabei unumstritten - das Problem ist allerdings, wie stark das geographische Milieu die Essenz einer Religion beeinflusst. Es besteht ein gewisser Konsens darüber, dass die Gestalt des Naturraumes das religiöse Verhalten zwar nicht formt, es dafür aber auf eine ganz besondere Weise färbt und ihm je nach geographischem Raum und Gesellschaft ein besonderes Lokalkolorit verleiht. ${ }^{22}$ Es wäre daher legitim zu vermuten, dass sich die Wirkung der Geofaktoren vordergründig im Bereich der Handlungsweisen (Riten) und nicht in dem der Glaubensvorstellungen entfaltet. ${ }^{23}$ Auf diese Prämisse stützen sich nachfolgende Überlegungen zu den Interdependenzen zwischen Religion und Raum in der minoischen Kultur.

2 I $\mathrm{Zu}$ einer sehr einleuchtenden Behandlung dieses Problems s. Gehlen, Welt und Ordnung, a.O. (Anm. I I) 67-75, der eine sehr vorsichtige Haltung gegenüber Theorien zur Umweltabhängigkeit der Religion einnimmt; s. ferner Rinschede, Religionsgeagraphie, a. $O$. (Anm. I 8) 81-102; M. Schwind, „Einleitung: Über die Aufgaben der Religionsgeographie“, in: Ders. (Hg.), Religionsgeographie (1975) 5-7. Zu einer überblickenden Darstellung über die Entstehung und Entwicklung derartiger religionsgeographischer Vorstellungen von der Antike bis in die Gegenwart s. Rinschede, Religionsgeographie, a. O. (Anm. 18) 2427.

22 s. hierzu Rinschede, Religionsgeographie, a. O. (Anm. I8) 20. 89-91; Marinatos, Ritual, a.O. (Anm. 6) I I 5: „Expression of religious feeling is, to a great extent, induced and shaped by the natural environment."

$23 \mathrm{Zu}$ diesen beiden elementaren Kategorien religiöser Phänomene s. Durkheim, Die elementaren Formen, a. O. (Anm. 8) 6I. 


\section{Die symbolische Strukturierung des geographischen Realmilieus}

Wie wurde die spannungsgeladene Landschaft Kretas in minoischer Zeit erfahren und durch Bezug auf das Gesamtgefüge einer symbolischen Ordnung strukturiert? Als Leitbilder der imaginären räumlichen Ordnung in allen Perioden der kretischen Geschichte dienten zweifellos die Berge. Die Bedeutung des Berges als vertikaler Verankerung des Göttlichen ist eine religionsanthropologische Konstante. ${ }^{24}$ In allen Kulturen, deren Lebensraum von Gebirgen geprägt war, werden die Berge sakralisiert. Die Frage, warum die Vertikalachse eine so große Signifikanz in verschiedenen Religionen besitzt, ist von Theologen und Religionswissenschaftlern eingehend behandelt worden. Es gibt eine Reihe von geomorphologischen und kosmologischen Gründen, die zur Bergverehrung führen. Viel wichtiger als terrestrische und wahrnehmungspsychologische Gesichtspunkte, nämlich ihre große Höhe und Prägnanzform, deren Wirkungskraft durch die wechselnden Farben der Jahreszeiten (Schneekuppen oder Wolkenhüllen) verstärkt wird, ist dabei die kosmologische Vorstellung eines axis mundi, ${ }^{25}$ welcher die Erde mit dem Himmel verbindet und die Nähe zur göttlichen Sphäre ermöglicht. Entscheidend ist dabei, dass nach einer geographisch und zeitlich sehr verbreiteten Vorstellung die Götter im Himmel leben. ${ }^{26}$ Ihre Sakralität wurde auch mythologisch begründet, denn sie besaßen eine primordiale Bedeutung als Orte der ersten menschlichen

24 Die einzige umfassende ethnologische Studie zur Bergverehrung liegt bereits über ein Jahrhundert zurück, s. F.v. Andrian, Der Höhencultus asiatischer und eurapäischer Völker. Eine ethnologische Studie (1 891), bes. S. XIII-XXXIV; s. ferner F. Tichy, Die geordnete Welt indianischer Völker. Ein Beispiel, von Raumordnung und. Zeitordnung im vorkolumbianischen Mexiko (1991) 1 59-167; E. Bernbaum, Sacred Mountains of the World (1998) [dem Verf. nicht zugänglich]; A. Cooper, Sacred Mountains. Ancient. Wisdom and.Modern Meanings (1997). Zur kosmologischen und rituellen Bedeutung der Berge in verschiedenen Religionen s. Fickeler, Religionsgeographie 81-83; I. Hori, Folk Religion in Japan. Continuity and Change (1968) I 4I-179; P. Gerlitz, „Buddhisten in Shintoschreinen“, in: M. Büttner (Hg.), Miteinander, Nebeneinander, Gegeneinander. Vielfalt religiöser, ethnischer, kultureller Gruppen und deren Beziehung zueinander im gemeinsamen Lebensraum. Ein Beitrag zur Geographie der Geisteshaltung (1994) 69-79; Rinschede, Religionsgeographie, a. O. (Anm. 18) 83-84; Horden - Purcell, Corrupting Sea, a.O. (Anm. 20) 408-414. Zur Bedeutung der Höhendimension in der biblischen Religion s. Schart, „Die Entgrenzung des heiligen Raumes“, a.O. (Anm. 9) 349-350. Zur mythischen bzw. sakralen Dimension der Berge im antiken Griechenland s. R.G.A. Buxton, „Imaginary Greek Mountains“, Journal of Hellenic Studies 112 (1992) 6-I 5; ders., Imaginary Greece (1994) 85-86. 90-92.

25 Eliade, Das Heilige 103.

26 s.v. Andrian, Höhencultus, a.O. (Anm. 24) S. XVI: „Die an den Berggipfeln hervortretenden Lichterscheinungen, das wechselvolle Spiel der Wolken an den Höhen bezeugen gleichsam das innige Verhältnis der Berge zu dem Himmel. Dieser überirdische Charakter wird durch die Schwierigkeit der Annäherung, durch die über hohen Bergspitzen ausgegossene erhabene Ruhe noch verstärkt." 
Besiedlung und als Lebensraum, in dem die Götter ihre Jugend verbrachten. ${ }^{27}$ Ihre Entfernung von der urbanen Sphäre, ihre Abgeschiedenheit, das was Horden und Purcell sehr treffend als "frightening detachment from the normal conditions of life ${ }^{{ }{ }_{28}}$ bezeichneten, machen die Berge zu den wichtigsten Schauplätzen religiöser und mythischer Aktion. Die in der kretischen Landschaft sehr klar umrissenen Grenzen zwischen Flachtälern und Bergketten fassen die verschiedenen menschlichen Lebens- und Wirkungsbereiche sehr klar ein, unterscheiden den auf intensive Weise wirtschaftlich erschlossenen und bewohnbaren Lebensraum von der Wildnis der Berge und verstärken dadurch den Eindruck einer bedeutungsvollen räumlichen Differenzierung, einer primordial strukturierten Welt. Der Berganstieg ist auf Kreta immer eine ganz besondere Erfahrung, das Eindringen in eine andere Welt, gewesen. Es liegt daher nahe zu vermuten, dass sich auch auf Kreta der größte Teil des religiösen Geschehens in den Bergen abgespielt hat und dass diese Gebirgsräume die Regionen waren, die dichter mit sakralen Orten besetzt wurden. Die Ebenen gehörten offensichtlich größtenteils zum - im Sinne Eliades - amorphen, symbolisch nur schwach besetzten und strukturlosen Teil des erlebten Raumes, eine ,profane Provinz', die sich vor allem nicht durch Ritualaktion, sondern durch „zweckrational und technologisch motiviertes Handeln ${ }^{{ }_{29}}$ zur Bewältigung des Alltags auszeichnete.

Als eine Versinnbildlichung der Sakralität der kretischen Berge oder eines bestimmten kretischen Berges in minoischer Zeit könnte ein neupalastzeitlicher Siegelring betrachtet werden, von dem nur seine Abdrücke aus dem Palast von Knossos bekannt sind: Eine Göttin (Mother of the Mountain) steht auf einem kegelförmigen Steingebilde - offensichtlich einem Hügel oder Berg -, an deren beiden Seiten sich je ein Löwe mit den Pfoten aufstützt (Abb. 2).$^{30}$ In einer ähnlichen Darstellung (Master Impression) sehen wir eine männliche Gestalt in einem gebieterischen Gestus, die auf einem Gebäude steht, welches ebenfalls auf einem Hügel bzw. einer Felsstruktur errichtet ist. ${ }^{31}$ Auch wenn die Entzifferung der hier entfalte-

27 s. R. G. A. Buxton, Imaginary Greece (1994) 90.

28 Horden - Purcell, Corrupting Sea, a. O. (Anm. 20) 408; s. ferner R. G. A. Buxton, „Imaginary Greek Mountains“, a.O. (Anm. 24) 15: „Mountains are unsettling, for those in settlements; they are to be viewed from afar, visited only to be left again“.

29 Pongratz-Leisten, Ina šulmi irub, a. O. (Anm. 5) I4. Diese Zweiteilung des von Menschen angeeigneten physischen Raumes wird dadurch augenscheinlicher, dass heilige Berge oft tabuisiert wurden, indem man ihr Betreten und ihre wirtschaftliche Nutzung durch rituelle Vorschriften regelte, s. v. Andrian, Höhencultus, a. O. (Anm. 24) S. XVII.

30 s. M.A.V. Gill - W. Müller - I Pini, Corpus der minoischen und mykenischen Siegel II 8. Iraklion, Archäologisches Museum. Die Siegelabdrücke von Knossos (2002) Nr. 256 (mit ausführlichen Literaturangaben).

3 I E. Hallager, The Master Impression. A Clay Sealing from the Greek-Swedish Excavations at Kastelli, Khania. Studies in Mediterranean Archaeology 69 (1985); I. Pini (Hg.), Corpus der minoischen undimykenischen Siegel V, Suppl. I A (1992) Nr. 142. 
ten symbolischen Sprache keine leichte Angelegenheit ist, darf eine Verbindung des Göttlichen mit dem Berg oder zumindest mit der Höhendimension als die plausibelste Interpretation der intendierten semantischen Botschaft beider Bilder gelten. Einen unmissverständlichen Beleg dieser Verbindung finden wir in der späteren griechischen Überlieferung, die um die Person des mythischen Königs Minos kreist. Alle neun Jahre, im ,Großen Jahr', suchte

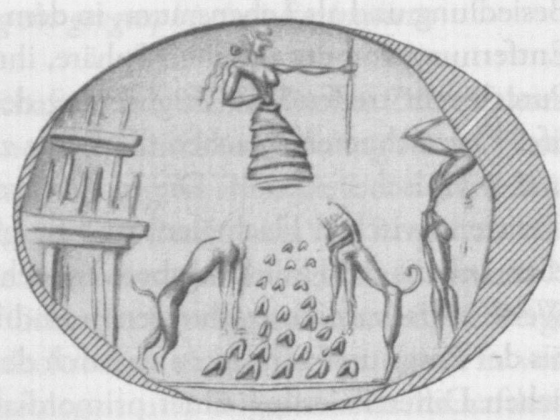

Abb. 2: , Mother of the Mountain': Siegelabdruck aus dem Palast von Knossos. Minos die Höhle und Geburtsstätte des kretischen Zeus in der Nida-Hochebene auf, um dort, in der Abgeschiedenheit der Berge, wie ein zweiter Moses seinem Gott zu begegnen und von ihm belehrt zu werden. ${ }^{32}$ Es kann keinen Zweifel daran geben, dass die Idäische Zeus-Grotte und die Nida-Hochebene, eine ganz besondere Landschaft, I538 m über dem Meeresspiegel, einen der Mittelpunkte der imaginären Kartographie der frühkretischen Religion im I. Jt. v. Chr. bildete. ${ }^{33}$ Dass die Idäische Grotte diese Bedeutung bereits in minoischer Zeit erlangt hatte, lässt sich durch einige Funde mit religiösen Konnotationen plausibel machen. ${ }^{34}$ Doch gibt es einen anderen Berg, der - obwohl kleiner als der Ida - wegen seiner Lage und Form als der minoische heilige Berg geradezu prädestiniert war. Es handelt sich um den Jouchtas-Berg, der in der unmittelbaren Nähe und in Sichtweite des Palastes von Knossos lag und eine sehr markante geographische Erhebung bildete (Abb. 3). Es ist sicherlich kein Zufall, dass auf dessen Gipfel eines der wichtigsten minoischen Höhenheiligtümer lag. ${ }^{35}$

32 s. hierzu H. Verbruggen, Le Zeus crétois (I981) 84-85, mit Verweisen auf die einschlägigen antiken Quellen.

33 s. zuletzt K. Sporn, Heiligtümer und Kulte Kretas in klassischer und hellenistischer Zeit. Studien zu Antiken Heiligtümern 3 (2002) 21 8-223 (mit ausführlichen Literaturangaben).

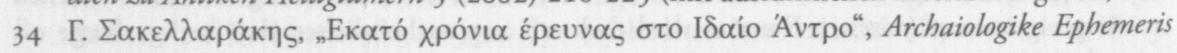
(1987) 247.

35 A. Karetsou, „The Peak Sanctuary of Mt. Juktas“, in: R. Hägg - N. Marinatos (Hgg.), Sanctuaries and Cults in the Aegean Bronze Age. Proceedings of the First International Symposium at the Swedish Institute in Athens, I2-13 May I980. Acta Instituti Atheniensis regni Sueciae 4, 28 (198I) I 37-I 53. Erwähnenswert ist in diesem Zusammenhang die Überlieferung vom Berg Jouchtas als Grabstätte des kretischen Zeus, die sich allerdings nicht bis in die Antike verfolgen lässt, s. hierzu Sakellarakis - Sakellarakis, Archanes, a. O. (Anm. 2) 50-5I; Verbruggen, Zeus Crétois, a. O. (Anm. 32) 63-67. 
Wie wurde innerhalb dieser gebirgigen Regionen der Landschaftsraum religiös und rituell besetzt? Welche waren die Fixpunkte der transzendentalen Erfahrung der Minoer in der Abgeschiedenheit der Berge? Die Entstehung sakraler Orte im kretischen Gebirgsraum scheint vor allem von zwei Faktoren abhängig gemacht worden zu sein: a) der besonderen räumlichen Spannungskapazität und b) der Liminalität bzw. dem Brückencharakter zwischen zwei verschiedenen Sphären. Der erste Parameter bezieht sich auf die kognitive Raumwahrnehmung, der zweite auf die Verschränkung zwischen religiösen Vorstellungen und sozio-ökonomischer Realität. Die Landschaftsphysiognomie, die Lage und vor allem die besondere atmosphärische Wirkung einer Lokalität, die nicht nur Staunen

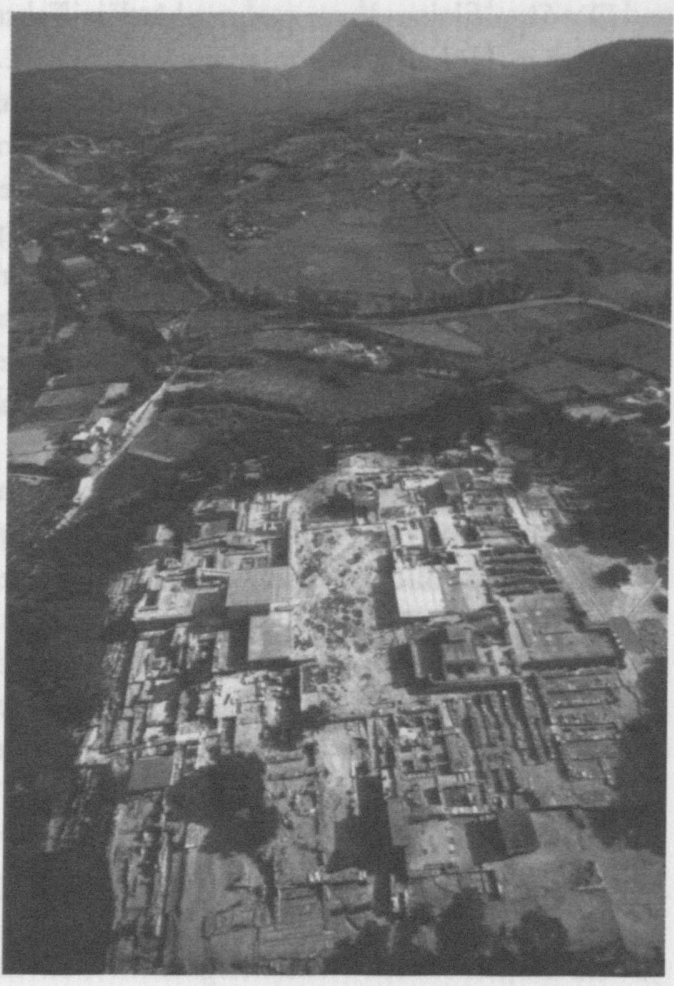

Abb. 3: Der minoische Palast von Knossos (von N). Im Hintergrund der Berg Jouchtas. hervorriefen, sondern auch die

Präsenz oder das Wirken des Numinosen nahe legten, waren sicherlich, wie bereits erwähnt, wichtige Faktoren für die Sakralisierung eines Ortes. Von besonderem Interesse ist allerdings die Tatsache, dass sich die sakralen Orte in den kretischen Bergen immer innerhalb einer Zone befanden, die dem Radius menschlicher Aktivität entsprach und daher nicht schwer zugänglich war - und damit kommen wir zum zweiten Parameter, dem der Liminalität. Der sakrale Ort als Schnittstelle zwischen der menschlichen und göttlichen Sphäre musste in einer realgeographischen oder imaginären Randzone gelegen sein, die für die Menschen leicht zugänglich oder zumindest gut sichtbar war. Aus diesem Grund wurden die schwer erreichbaren Gipfel der kretischen Bergketten, d.h. ihre markantesten geographischen Punkte, offensichtlich nie zu wichtigen sakralen Orten überhöht, weder in minoischer Zeit noch in späteren Perioden. Der Grund ist, dass sie entweder schwer zugänglich oder von den bewohnten Regionen aus nicht sichtbar waren. 
Die Signifikanz dieser geographischen Faktoren lässt sich sehr gut im Fall der minoischen Höhenheiligtümer nachvollziehen. Sie stellen den wichtigsten Typus eines minoischen Heiligtums dar, den man archäologisch gut fassen kann. ${ }^{36}$ Solche Höhenheiligtümer sind auf der ganzen Insel verstreut und zeigen in ihrer überwiegenden Mehrheit eine minimale bauliche Ausgestaltung - sie werden nicht einmal durch eine Temenos-Mauer vom profanen homogenen Raum ausgegrenzt. Auch in den wenigen Höhenheiligtümern, die mit einfachen Bauten ausgestattet waren, fanden die Kultaktivitäten offensichtlich im Freien statt. Diese sakralen Orte lagen - bis auf vereinzelte Ausnahmen - nicht auf den höchsten Gipfeln der kretischen Bergketten, sondern auf niedriger gelegenen Anhöhen, die vor allem folgende Voraussetzungen erfüllten mussten:

a) prominente Lage (auffallende, dominante, weithin sichtbare Gipfel oder Anhöhen),

b) Visibilität (Blickkontakt zu einer oder mehreren Siedlungen oder zu einem oder mehreren Höhenheiligtümern),

c) leichter Zugang von den benachbarten Siedlungen aus,

d) Nähe zu Acker-oder Weideland und somit zur Lebensgrundlage der lokalen Bevölkerung. ${ }^{37}$

Es wird dadurch ersichtlich, dass zur Auswahl des heiligen Ortes keine kosmologische, sondern vor allem wahrnehmungspsychologische oder ganz pragmatische Überlegungen ausschlaggebend waren. ${ }^{38}$ Die symbolische Strukturierung der kreti-

36 Zu den minoischen Höhenheiligtümern s. B. Rutkowski, Cult Places in the Aegean World (1972) 152-188; A. D. Peatfield, "The Topography of Minoan Peak Sanctuaries“, The Annual of the British School at Athens 78 (1983) 273-280; ders., „Minoan Peak Sanctuaries: History and Society“, Opuscula Atheniensia 17 (1990) $117-131$; ders., "Rural Ritual in Bronze Age Crete: The Peak Sanctuary at Atsipadhes“, Cambridge Archaeological Journal 2 ( 992 ) 59-87; ders., "Divinity and Performance on Minoan Peak Sanctuaries", in: R. Laffineur - R. Hägg (Hgg.), POTNIA. Deities and Religion in the Aegean Bronze Age. Proceedings of the 8th International Aegean Conference, Göteborg University 12-15 April 2000. Aegaeum 22 (2001) 5 I-55; L. V. Watrous, "Some Observations on Minoan Peak Sanctuaries", in: R. Laffineur - W.-D. Niemeier (Hgg.), POLITEIA. Society and State in the Aegean Bronze Age. Proceedings of the sth International Aegean Conference, University of Heidelberg, Archäologisches Institut, 10-13 April 1994. Aegaeum 12 (1995) 393-402; K. Nowicki, "Minoan Peak Sanctuaries: Reassessing their Origins", in: Laffineur - Hägg, a. O. 3 1-36; E. Kyriakidis, Ritual in the Bronze Age Aegean. The Minoan Peak Sanctuaries (2005).

$37 \mathrm{Zu}$ diesen vier wichtigen Faktoren zur Ortsauswahl eines minoischen Höhenheiligtums s. A. D. Peatfield, "Rural Ritual in Bronze Age Crete: The Peak Sanctuary at Atsipadhes“, Cambridge Archaeological Journal 2 (1992) 60.

38 Dass die Visibilität und die Höhe über der sichtbaren Umgebung wichtiger als die absolute Höhe sind, unterstreicht auch P. Fickeler, Religionsgeographie 82. Zu einer interessanten Fallstudie zur sakralen Topographie des neuzeitlichen und modernen Kreta s. L. Nixon, Making a Landscape Sacred. Outlying Churches and. Icon Stands in Sphakia, Southwestern Crete (2006) bes. 7-1 3. 92-I I6. 
schen Landschaft war also nicht nur religiösen, sondern auch sozio-ökonomischen Ordnungsprinzipien unterworfen. ${ }^{39}$

Neben den Höhenheiligtümern boten Höhlen einen bevorzugten Ort kultischer Aktivität. ${ }^{40}$ Höhlen besitzen von Natur aus einen liminalen Charakter und können daher als Schnittstellen zwischen der realen und der transzendentalen Welt fungieren. ${ }^{41}$ Der Abstieg in eine große Kulthöhle war sicherlich eine besondere Erfahrung. Kein gebauter Tempel kann suggestiver wirken als ein dunkler, feuchter, unterirdischer Raum, dessen tief gelegenen Kultplatz man durch einen engen, steil absteigenden Korridor erreichte. Stalagmiten, die eine menschen- oder tierähnliche Form aufwiesen, erhielten offensichtlich eine religiöse oder kultische Bedeutung als göttliche Erscheinungen und wurden entweder innerhalb einer Höhle durch Weihungen markiert oder durch eine Temenos-Mauer hervorgehoben. In der Psychro-Kulthöhle waren Doppeläxte als Votive oder sakrale Markierungen in die Stalagmiten eingetieft..$^{22}$ In der Eileithyia-Höhle in Amnissos waren zwei Stalagmiten vielleicht bereits in minoischer Zeit durch eine kleine Temenos-Mauer eingegrenzt. ${ }^{43}$

Das nach unserem jetzigen Kenntnisstand bedeutendste eigenständige $-d$. $h$. nicht in einen profanen architektonischen Komplex eingebettete - minoische Hei-

39 s. hierzu L. V. Watrous, The Cave Sanctuary of Zeus at Psychro. A Study of Extra-urban Sanctuaries in Minoan and Early Iron Age Crete. Aegaeum is (1996) 75-8I; A. Peatfield, „After the ,Big Bang“ - What? or Minoan Symbol and Shrines beyond Palatial Collapse", in: S. E. Alcock - R. Osborne (Hgg.), Placing the Gods. Sanctuaries and Sacred Space in Ancient Greece (1994) 23. Interessanterweise haben ähnliche Überlegungen die Auswahl sakraler Orte auf Kreta auch in späteren Perioden, sogar bis in die Gegenwart, geleitet. Die neuzeitlichen oder modernen kleinen Kapellen, die überall auf der Insel verstreut sind, fehlen merkwürdigerweise in den hohen, schwer zugänglichen und ökonomisch uninteressanten Regionen des Psiloritis und der Weißen Berge. In Madares, einer kahlen und im Winter unzugänglichen Region der Weißen Berge, gibt es keine einzige Kapelle, s. Nixon, Making. a Landscape Sacred, a. O. (Anm. 38) 88-89.

$40 \mathrm{Zu}$ kretischen Höhlen als Kultstätten in minoischer Zeit s. Rutkowski, Cult Places, a. O. (Anm. 36) I 2 I-I 5 I; Marinatos, Ritual, a. O. (Anm. 6) I 23-1 26; Watrous, Cave Sanctuary, a. O. (Anm. 39) $57-63$.

4I Zu Höhlen als Schnittstellen zwischen der menschlichen und der göttlichen Sphäre, s. E. L. Tyree, „Diachronic Changes in Minoan Cave Cult", in: Laffineur - Hägg, POTNLA, a. O. (Anm. 36) 40. Zur mythischen und kultischen Bedeutung von Höhlen im antiken Griechenland s. R. G. A. Buxton, Imaginary Greece (1994) I04-I 08.

42 Rutkowski, Cult Places, a.O. (Anm. 36) I 3 I-132. Zum minoischen Kult in Psychro s. Watrous, Cave Sanctuary, a.O. (Anm. 39) 47-53. Zur kultischen Bedeutung von Stalag-

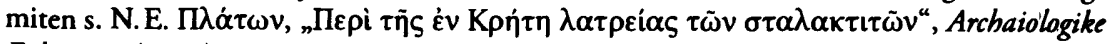
Ephemeris (1930) I60-168.

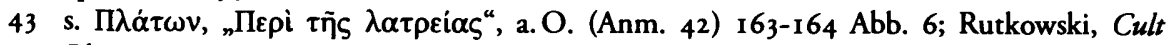
Places, a. O. (Anm. 36) I 29-1 30. 
ligtum befindet sich in Symi Viannou (Ost-Kreta). ${ }^{44}$ Die Kultterrasse und der/die Kultgebäude der minoischen Benutzungsphase liegen unterhalb jüngerer Schichten aus dem I. Jt. v. Chr., als dieses Quellheiligtum als Kultstätte von Hermes und Aphrodite besondere Bedeutung erlangte.45 An dieser Stelle muss betont werden, dass dieses eindrucksvolle Bild einer Kultkontinuität keine Regel, sondern eher die Ausnahme darstellt. Es ist sicherlich merkwürdig, dass es auf fast keinem einzigen minoischen Höhenheiligtum Anzeichen eines Kultes nach dem Ende der minoischen Ära gibt. ${ }^{46}$ Die einzige plausible Erklärung für diesen Umstand wäre die vorhin angesprochene Bedeutung der sozio-ökonomischen Aspekte des Kultes, wonach die Auswahl der Kultplätze nicht nur von religiösen oder kosmologischen Überlegungen, sondern auch von den Besiedlungsmustern determiniert wurde.

\section{Sakrale (Tat-)Orte}

Werfen wir nun einen Blick auf das Individuum und die Interaktion zwischen Mensch und Raum innerhalb einer sakralen Sphäre. Die Bilderwelt belehrt uns, dass die wichtigsten Zeremonien der minoischen Religion stets in eine landschaftliche Umgebung eingebettet waren und im Freien, in einigen Fällen vor einem Kultbau oder Schrein, stattfanden. ${ }^{47}$ Auf einem reliefierten Steingefäß aus Knossos wird die Kulisse dieser Rituale mit semantisch sehr klaren Mitteln ins Bild gesetzt: ${ }^{48}$ Wir sehen eine Temenos-Mauer, die den Bereich des Heiligtums abgrenzt, in dessen Mitte ein Altar steht (Abb. 4). Im Hintergrund erscheint ein Baum, der vielleicht in einem inneren Bereich des Heiligtums - vermutlich einem heiligen Hain - lag.

Im Mittelpunkt dieser Kultpraxis stand ein ekstatisches Ritual, bei dem Adoranten bzw. Priester als Hauptakteure aufgetreten sind. ${ }^{49}$ Die hier stattfindende Ritualaktion hatte drei Bestandteile: das kräftige Schütteln eines Baums, einen ekstatischen Tanz oder kräftiges Schwingen des Körpers sowie das Berühren, Umar-

44 s.o. Anm. 3; ferner Watrous, Cave Sanctuary, a. O. (Anm. 39) 65-70.

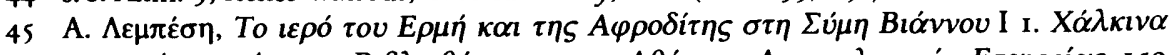

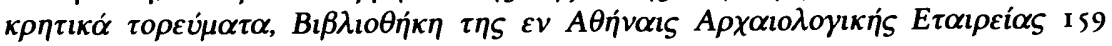
(1985).

46 Nixon, Making a Landscape Sacred, a. O. (Anm. 38) I 44 Anm. 137.

47 P. Warren, Minoan Religion as Ritual Action (1988) 33.

48 A. Evans, The Palace of Minos at Knossos II (1928) 61 4-6 I6 Abb. 386.

49 Tyree, „Diachronic Changes“, a. O. (Anm. 4I) 42; Marinatos, Ritual, a. O. (Anm. 6) I75188. $\mathrm{Zu}$ einer umfassenden und konzisen Studie der religiösen Ekstase s. I.M. Lewis, An Anthrapologic Study of Spirit Possession and Shamanism (I 971 ). 
men bzw. Küssen eines Steinmals (Baitylos)..$^{\circ}$ Die scheinbar unkontrollierbaren Bewegungen der Personen, die an diesen Handlungen beteiligt waren, versinnbildlichen den orgiastischen Charakter der religiösen Erfahrung (Abb. 5). ${ }^{\text {sI }}$ Es herrscht allgemeiner Konsens darüber, dass der Sinn des dargestellten Rituals das Heraufbeschwören der göttlichen Erscheinung war. ${ }^{52}$ In einigen dieser Szenen steigt tatsächlich die Gottheit - in kleinerem Format dargestellt - aus dem Himmel herab und wird offensichtlich von den in einem Trance-Zustand befindlichen Akteuren des Rituals visuell wahrgenommen.53 Die bildliche Umsetzung dieses EpiphanieRituals ${ }^{54}$ ist uns in verschiedenen Varianten - mit oder ohne göttliche Erscheinung, mit einer oder allen drei rituellen Handlungen - bekannt." Wie das Ritual tatsächlich abgelaufen ist und wie die Kultgemeinde an der Epiphanie der Gottheit

so Zu den Baityloi s. Warren, Minoan Religion, a.O. (Anm. 47) 16-18; ders., „Of Bae-

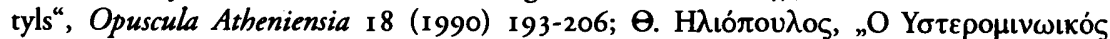

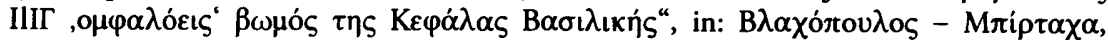

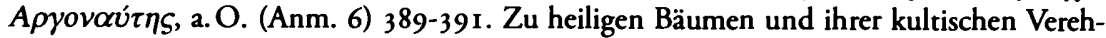
rung s. M.P. Nilsson, The Minoan-Mycenaean Religion and its Survival in Greek Religion ${ }^{2}$ (1950) 262-288; B. Rutkowski, „Der Baumkult in der Ägäis“, Visible Religion 3 (1984) I 59-165; Eliade, Das Heilige I 3 I; N. Marinatos, „The Tree as a Focus of Ritual Action in Minoan Glyptic Art“, in: W: Müller (Hg.), Fragen und Probleme der bronzezeitlichen ägäischen Glyptik. Beiträge zum 3. Internationalen Marburger Siegel-Symposium, 5.-7. September 1985. Corpus minoischer und mykenischer Siegel; Beih. 3 (1989) 1 27-143; W: Pötscher, Aspekte und Probleme der minoischen Religion. Ein Versuch (1990) 80.

5 I „[...] con furore orgiastico“ wie es L. Savignoni treffend ausdrückte, s. L. Savignoni, „Scavi e scoperte nella necropoli di Phaestos“, Monumenti antichi I4 (1904) 578 (zitiert in Warren, Minoan Religion, a. O. [Anm. 47] i6).

52 Nilsson, Minoan-Mycenaean Religion, a.O. (Anm. 5o) 277-283; R. Hägg, „Die göttliche Epiphanie im minoischen Ritual“, Mitteilungen des Deutschen Archäologischen Instituts, Athenische Abteilung. IOI (1986) 46. 62; A. Evans, The Palace of Minos at Knossos III (1930) 69-72. I40; Marinatos, Ritual, a.O. (Anm. 6) I75; B. Gladigow, „Epiphanie, Statuette, Kultbild. Griechische Gottesvorstellungen im Wechsel von Kontext und Medium“, Visible Religion 7 I (1990) 104.

53 Zum Epiphanie-Gestus der am Ritualgeschehen beteiligten Personen s. Gladigow, „Epiphanie“, a. O. (Anm. 52) 100. I02; zur spiegelbildlichen Symmetrie des Gestus der Göttin und der Kultpersonen s. auch E. Brandt, Gruß und Gebet. Eine Studie zu Gebärden in der minoisch-mykenischen und frühgriechischen Kunst. (1965) 6-10.

54 Hier soll angemerkt werden, dass der von Eliade bevorzugte Begriff ,Hierophanie', die „von Menschen wahrnehmbare oder empfundene Manifestation des Heiligen“, eine bessere Alternative als das semantisch neutrale Wort ,Epiphanie‘ bietet, s. Eliade, Das Heilige I4.

55 Das bekannteste Beispiel dieser Epiphanie-Szenen stellt der Goldring aus dem Kammergrab von Isopata dar. Zu dieser Darstellung und den damit verbundenen Interpretationsproblemen s. C. D. Cain, „Dancing in the Dark: Deconstructing a Narrative of Epiphany on the Isopata Ring", American Journal of Archaeology I0s (2001) 27-49. Trotz Cains berechtigter Kritik an bisherigen Versuchen, die narrative Struktur dieser Szene zu rekonstruieren, bleibt die traditionelle Deutung als göttliche Epiphanie die plausibelste. 
- an diesem Einbruch des Heiligen in die wahrnehmbare Welt - teilhatte, lässt sich natürlich aus diesen kursorischen Darstellungen nicht sagen. Man darf allerdings vermuten, dass sich auch die passiven Teilnehmer dieses ekstatischen Ritualdramas seiner enormen suggestiven Kraft nicht entziehen konnten. Die offensichtlich echte Ekstase $^{56}$ der Akteure war genug, um die Mitglieder der beiwohnenden religiösen Gemeinschaft zu überzeugen, dass sich die göttliche Epiphanie gerade vor ihren Augen vollzog, auch wenn sie von ihnen visuell nicht wahrgenommen werden konnte. ${ }^{57}$

Wenn wir diese Szenen als zentrale Bildmanifestationen der minoischen Kultpraxis betrachten möchten, können wir einige Hypothesen über die besondere Essenz des minoischen religiösen Glaubens aufstellen. Das, was

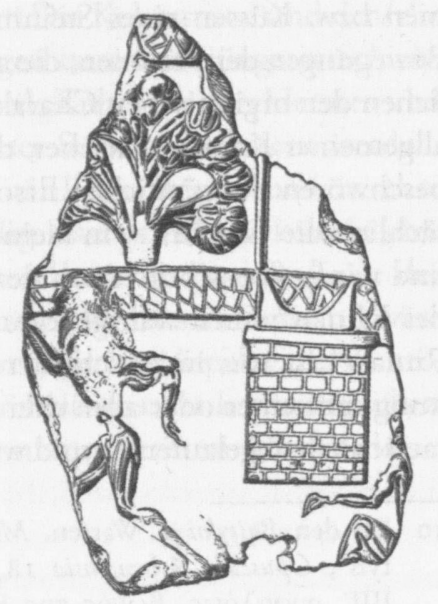

Abb. 4: Fragment eines reliefierten Steingefäßes mit Darstellung eines Altars im umfriedeten Bezirk eines ,Temenos: diese Bilder implizieren, ist eine Religion, die mit den stark theozentrischen jüdischen und christlichen Glaubensvorstellungen wenig gemeinsam hat, eine Religion, in der schamanistische oder orgiastische Elemente überwiegen. ${ }^{58}$ Nicht der Gott und die aus Beten, Flehen und Weihen geprägte Kultpraxis anderer Religionen stehen hier im Mittelpunkt, sondern Ritualhandlungen, bei denen der menschliche Körper das Medium der transzendentalen Erfahrung, ein Kanal der einverleibten Wahr-

56 Anstelle des Begriffs, Ekstase ' bevorzugen Chr. Morris und A. Peatfield den neutraleren Terminus altered state of consciousness, s. Chr. Morris - A. Peatfield, „Feeling through the Body. Gesture in Cretan Bronze Age Religion“, in: Y. Hamilakis - M. Pluciennik - S. Tarlow (Hgg.), Thinking through the Body. Archaeologies of Corporeality (2002) I I0; s. hierzu auch C. T. Tart (Hg.), Altered States of Consciousness (1969).

57 s. hierzu auch F. Matz, Göttererscheinung und Kultbild im minoischen Kreta. Akademie der Wissenschaften und der Literatur in Mainz. Abhandlungen der Geistes- und Sozialwissenschaftlichen Klasse 7 (1958) 422; ferner Marinatos, Ritual, a. O. (Anm. 6) 178. Entscheidend ist aus soziologischer Sicht nicht der Trance-Zustand des Einzelnen, sondern die religiöse Exaltation der dem Ritual beiwohnenden Gruppe, s. Lewis, Anthropologic Study, a. O. (Anm. 49) 64-65.

58 Diese orgiastischen oder schamanistischen Aspekte der minoischen Religion, die bereits von A. Evans und seiner Generation erkannt wurden, rückten erst in den letzten Jahren wieder in den Mittelpunkt der wissenschaftlichen Aufmerksamkeit, s. A. Peatfield, „Divinity and Performance on Minoan Peak Sanctuaries", in: Laffineur - Hägg, POTNIA, a. O. (Anm. 36) 54-55. 
nehmung des Numinosen, war. ${ }^{59}$ Es wäre sicherlich anmaßend, zu glauben, dass wir mit diesen ekstatischen Ritualen das Wesen der minoischen Religion vollständig erfassen können. Diese Szenen geben uns Einblick auf nur einen Aspekt der minoischen Religion. Doch scheint dieser Aspekt, wenn man von den Bildträgern ausgeht (Goldringe, d.h. Insignien der palatialen oder priesterlichen Elite), recht bedeutend gewesen zu sein. In diesem Zusammenhang ist der Tanz als Ritualaktion von entscheidender Bedeu-

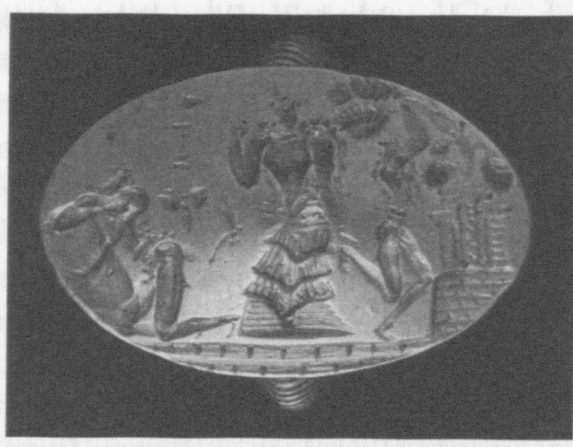

Abb. 5: Goldring aus dem Tholosgrab A von Phourni bei Archanes mit der Darstellung eines orgiastischen Rituals. tung, wenn es uns darum geht, das Dreieck ,sakraler Ort - Mensch - religiöse Erfahrung' greifbar zu machen. Einen Schlüssel zum Verständnis der Ereigniskette Tanz - Ekstase - Epiphanie bietet ein älterer Aufsatz von E. Straus, dem O. F. Bollnow in seiner - immer noch grundlegenden - phänomenologischen Studie „Mensch und Raum“ besondere Beachtung schenkte. ${ }^{60}$ Straus unternahm hier eine raum- bzw. körperbezogene Auslegung des Tanzes und ging von der Prämisse aus, dass sich Zweckbewegung und Tanz als zwei Bewegungsformen grundsätzlich voneinander unterscheiden, da sie „auf zwei ganz

59 s. Marinatos, Ritual, a. O. (Anm. 6) 178. Zum enthusiastischen Charakter des minoischen Glaubens s. Warren, Minoan Religion, a.O. (Anm. 47) 36: „Religion was not the opium of the Minoan people. Rather, practicing their rituals among stalagmites and dark pools within the earth, or in built subterranean chambers, on windy mountain peak or mountain side, in rural temple or palace or house or urban shrine, the Minoans display a positive, enquiring response, a proto-European attempt to understand and explain their environment and their cosmos." Dies hat bereits Matz unterstrichen: „Es ist ein enthusiastischer Glaube. Schon an den ekstatischen Formen des Kultes sieht man es [...] Man hat angesichts der minoischen Bilderwelt überhaupt und schon ohne auf ihre Formstruktur zurückzugreifen den Eindruck eines von Begeisterung und vibrierendem Temperament erfüllten und getriebenen menschlichen Wesens", s. Matz, Göttererscheinung, a.O. (Anm. 57) 447. Folgerichtig interpretieren C. Morris und A. Peatfield Gestik und Haltung der Tonstatuetten der minoischen Gipfelheiligtümer nicht als Anbetungs-, sondern als ekstatische Gesten, s. Morris - Peatfield, „Feeling through the Body“, a.O. (Anm. 56) I05-I I6. Auch diese Interpretationsmöglichkeit war Matz, Göttererscheinung, a. O. (Anm. 57) 408 nicht entgangen.

60 E. Straus, „Die Formen des Räumlichen. Ihre Bedeutung für die Motorik und die Wahrnehmung", in: Ders., Psychologie der menschlichen Welt. Gesammelte Schriften (1960) I4II78; s. ferner Bollnow, Mensch und Raum 244-256. 
verschiedene Modi des Räumlichen bezogen sind“" ${ }^{61}$ Er unterstrich, dass man mit dem Tanz ein verändertes Verhältnis zum Raum gewinne: „Beim Gehen bewegen wir uns durch den Raum, von einem Ort zum andern, beim Tanzen bewegen wir uns im Raum. "62 In diesem durch das Tanzen erschaffenen und erlebten Raum, der sich vom alltäglichen Zweckraum absetzt, vollzieht sich die „Aufhebung der zwischen Subjekt und Objekt, Ich und Welt bestehenden Spannung“ - der Mensch wird selber gewissermaßen ein Teil dieses Raums. ${ }^{{ }_{3}}$ Eine weitere Beobachtung von Straus ist schließlich im Kontext der minoischen ekstatischen Rituale von besonderen Bedeutung: „Dass die Tanzbewegung keine zeitliche Grenze kennt, dass sie erst durch die Erschöpfung oder Ekstase beendet wird, das ist überall zu beobachten, wo der Tanz noch nicht zum Gesellschafts- oder Kunsttanz geworden ist. " ${ }_{44}$ Der aus räumlicher Sicht zweckfreie Tanz, diese in B. Waldenfels' Worten "Suspendierung von Bewegungszielen und Bewegungsumständen",6s trägt als Medium eines veränderten Verhältnisses zum Raum das Potential einer tiefen metaphysischen Erfahrung. ${ }^{66}$ Diese religiöse Sinndimension des Tanzens wäre mit dem oben erläuterten Charakter der minoischen heiligen Räume als Stimmungsräume, die ihre Sakralität der atmosphärischen Wirkung der von Menschen wahrgenommen Natur verdankten, völlig kongruent. Der ekstatische Tanz als Entrücken von der alltäglich-praktischen Welt des zweckhaften Handelns, als tiefe metaphysische Erfahrung, war herausgehoben aus dem historischen Geschehen ${ }^{67}$ und zielte auf die Vereinigung des Menschen mit dem Raum. Diese nachvollziehbare körperliche und mentale Erfahrung dürfte man als reale Grundlage des minoischen Epiphanie-Rituals betrachten: Raumwahrnehmung als Ausgangspunkt des Sakralen, zweckfreies Bewegen in diesem Raum mit dem Ziel, eins mit diesem Raum zu werden, ekstatische Erfahrung als Höhepunkt dieser Erfahrung, die schließlich in der visionären Erscheinung des Göttlichen gipfelte. Es ist vielleicht überflüssig auf den krassen Gegensatz hinzuweisen zwischen diesem ekstatischen Ritual, dessen Potenz in leiblich ergreifenden Gefühlen besteht, und einer kultischen Prozession,

6r Straus, „Formen des Räumlichen“, a.O. (Anm. 60) 160; s. hierzu auch B. Waldenfels, "Sichbewegen“, in: G. Brandstetter - Chr. Wulf (Hgg.), Tanz als Anthropologie (2007) 28: „Der Tanz lebt von den Überschüssen einer Beweglichkeit, die sich nicht in Zwecken und Regeln fassen lässt. Er ist zweck- und regellos, gemessen an den Zwecken und Regeln des gewöhnlichen Lebens."

62 Straus, „Formen des Räumlichen“, a.O. (Anm. 60) I64.

63 Bollnow, Mensch und Raum 252. Wenn der Mensch eins mit dem Raum wird, lässt sich Natur nicht als ,Umwelt', sondern als,Mitwelt ${ }^{\star}$ - eine in vielen vormodernen Gesellschaft übliche Vorstellung - begreifen.

64 Straus „Formen des Räumlichen“, a. O. (Anm. 60) 164.

65 Waldenfels, "Sichbewegen“, a.O. (Anm. 6r) 30.

66 s. auch Bollnow, Mensch und Raum, 248-254.

67 Straus, „Formen des Räumlichen“, a.O. (Anm. 60) nennt diese aus dem historischen Geschehen herausgehobene Zeitdimension ,präsentische Zeit". 
der Quintessenz einer eher nüchternen Beziehung zwischen Mensch und Gott, die ihren Sinn in der Versorgung des Letzteren durch Ersteren im Rahmen eines dout-des-Verhältnisses findet. Eine Prozession, das zweckgerichtete Fortbewegen mit der konkreten Absicht der Darbringung der Gaben, wäre im Rahmen dieser sehr schöpferischen Raumwahrnehmung in Naturräumen fehl am Platz.

\section{6. ,Miniaturlandschaften' undidie Relokalisierung des Naturkultes}

Es gibt eine Reihe von Indizien aus der kretischen Neupalastzeit, wonach die Durchführung der orgiastischen Riten und die damit verbundenen transzendentalen Erfahrungen nicht nur in der kretischen Wildnis möglich waren. Spätestens in der Neupalastzeit stellen wir fest, dass einige Elemente dieses sehr naturnahen Rituals ihre standörtliche Verbundenheit verloren und in die ,urbane' Landschaft der minoischen Siedlungszentren verpflanzt wurden. Steine und Stalagmiten wurden z. B. als Gegenstände religiöser Verehrung in offenen oder inneren Räumen einer Siedlung aufgestellt. ${ }^{68}$ Welche Rituale sich in diesen Räumen vollzogen und inwieweit diese Räume sakralisiert wurden, lässt sich allerdings nicht sagen. Die bloße Existenz eines Gegenstandes religiöser Verehrung ist sicherlich nicht ausreichend, um das unmittelbare räumliche Umfeld dieses Objekts als sakrale Sphäre zu deklarieren. Auf etwas sichererem Boden stehen wir in einem besonders interessanten Fall, dem des Zentralhofs des Palastes von Malia. Ein großer runder Stein, der zur Hälfte in die festgetretene Erde des Hofs eingetieft war, ist offensichtlich ein Baitylos, der interessanterweise nicht im Zentrum des Zentralhofs, sondern in gleicher Flucht mit einer Loggia am Westflügel des Palastes lag. ${ }^{69}$ Diese Loggia diente offensichtlich als Tribüne für die residierende Elite, die an dieser Stelle Ritualen und Zeremonien im Zentralhof des Palastes beiwohnte. Die Vermutung liegt daher nahe, dass sich das ursprünglich in Naturräumen angesiedelte ekstatische Ritual, das wir aus der Ikonographie kennen, auch innerhalb eines städtischen Kontextes, ja sogar innerhalb eines größeren architektonischen Komplexes vollzog.

In derselben Zeit, in der wir eine Übertragung von Elementen des orgiastischen Kultes auf die urbane Sphäre dingfest machen können, ergriff die Naturwelt Besitz von dem privaten oder semiprivaten Lebensraum der minoischen Elite. Zahlreiche Räume in Palästen und elitären Privathäusern wurden nämlich mit einem malerischen Raumdekor ausgestattet, der die Natur verherrlichte. In vielen Fällen handelt es sich um einen erdachten Biotop mit einer Fülle von Tieren, Vögeln und Pflanzen, die in der Natur nicht gemeinsam vorkommen, eine gemischte Flora

$68 \Pi \lambda \dot{\alpha} \tau \omega \nu,{ }_{n} \sigma \tau \alpha \lambda \alpha \kappa \tau \iota \tau \omega \dot{\omega} v^{\prime,}$, a. O. (Anm. 42) I60-163.

69 P. Warren, „Of Baetyls“, Opuscula Atheniensia 18 (1990) 203. 
und Fauna aus der Berg- und Flusswelt. ${ }^{\circ}$ In einigen Fällen wurden diese 'Traumlandschaften' mit einem Aktionsbild, offensichtlich einem Ritual, belebt. Hinter diesem sehr beliebten Darstellungstypus erkannten verschiedene Forscher - etwas voreilig - Bilder von sakralen Landschaften, eine Meinung, die sich hartnäckig bis heute hält. Doch sind eine rituelle Deutung der Bildthemen selbst, der Räume, in denen sie sich befinden, ja sogar des Mediums der Freskomalerei nichts anderes als methodisch unzulässige Verallgemeinerungen. Die Frage nach dem sakralen Charakter der dargestellten Natur muss in jedem einzelnen Fall separat und zwar durch einen kontextimmanenten Ansatz beantwortet werden. Nicht jede Landschaftsdarstellung im Raum eines Hauses oder Palastes muss religiös konnotiert gewesen sein. ${ }^{71}$ Daher empfiehlt es sich in unserem Zusammenhang, nur auf einige dieser Bilder zu fokussieren, für die ein sakraler Gehalt sehr wahrscheinlich erscheint. Dabei handelt es sich um eine kleine homogene Gruppe von Wandmalereien in Erdgeschossräumen von elitären Häusern auf Kreta und auf der benachbarten Insel Thera, deren kontextbezogene Betrachtung uns zunächst mit einem Paradoxon konfrontiert. Diese Räume, die nicht größer als 4 bis $6 \mathrm{~m}^{2}$ sind, wurden vollständig mit Naturszenen ausgemalt. ${ }^{72}$ Ihr besonderes Merkmal besteht darin, dass es sich dabei um fensterlose oder schlecht beleuchtete Kammern handelt, in denen die prächtigen Farben der Wandmalereien, ja sogar das Bildthema selbst nur mit der Hilfe von Kunstlicht visuell wahrgenommen werden konnte. Das beste kretische Beispiel bietet Raum 14 der Villa von Ajia Triada, der lediglich $1,60 \times 2,35 \mathrm{~m}$ misst (also 3,76 $\mathrm{m}^{2}$ groß ist) ${ }^{73}$. Drei Wände dieses fensterlosen Raumes waren vollständig ausgemalt, die vierte nahm zwei Türen eines Polythyron ein, von denen eine als Eingang diente. Die Beleuchtung dieses Raumes war nur mit Kunstlicht möglich gewesen, weil sich die schmale Tür gegen eine Vorhalle und nicht zu einem offenen Raum öffnete..$^{74}$ Die Darstellung nahm ursprünglich eine Höhe von ca. 2,20 m ein. Auf der O-Wand sehen wir eine sehr anmutige landschaftliche Szenerie mit reichem vegetabilem Dekor, in der eine fein bekleidete Frau barfuss in einer eigenartigen Haltung vor einer Mauer steht. Die Fresken der Seitenwände

70 s. Marinatos, Ritual, a. O. (Anm. 6) 193-196; s. ferner P. Schmitz-P.illmann, Landschaftselemente in der minoisch-mykenischen Wandmalerei. Winckelmann-Institut der. HumboldtUniversität zu Berlin 6 (2006).

71 s. A. Chapin, „A Re-Examination of the Floral Fresco from the Unexplored Mansion at Knossos“, Annual of the British School at Athens 92 (1997) I 2-20.

72 Zu einer Zusammenstellung und Diskussion der relevanten Befunde s. V. Stürmer, ,Naturkulträume' auf Kreta und Thera: Ausstattung, Definition und Funktion “, in: Laffineur - Hägg, POTNIA, a. O. (Anm. 36) 69-74.

73 P. Militello, Haghia Triada I. Gli Affreschi. Monografie della Scuola archeologica di Atene e delle missioni italiane in Oriente 9 (1998) 99-1 32. 250-282; Stürmer, „Naturkulträume““,

a. O. (Anm. 72) 69-7I.

74 Stürmer, „Naturkulträume“ ${ }^{\text {“ }}$, a. O. (Anm. 72) 71. 


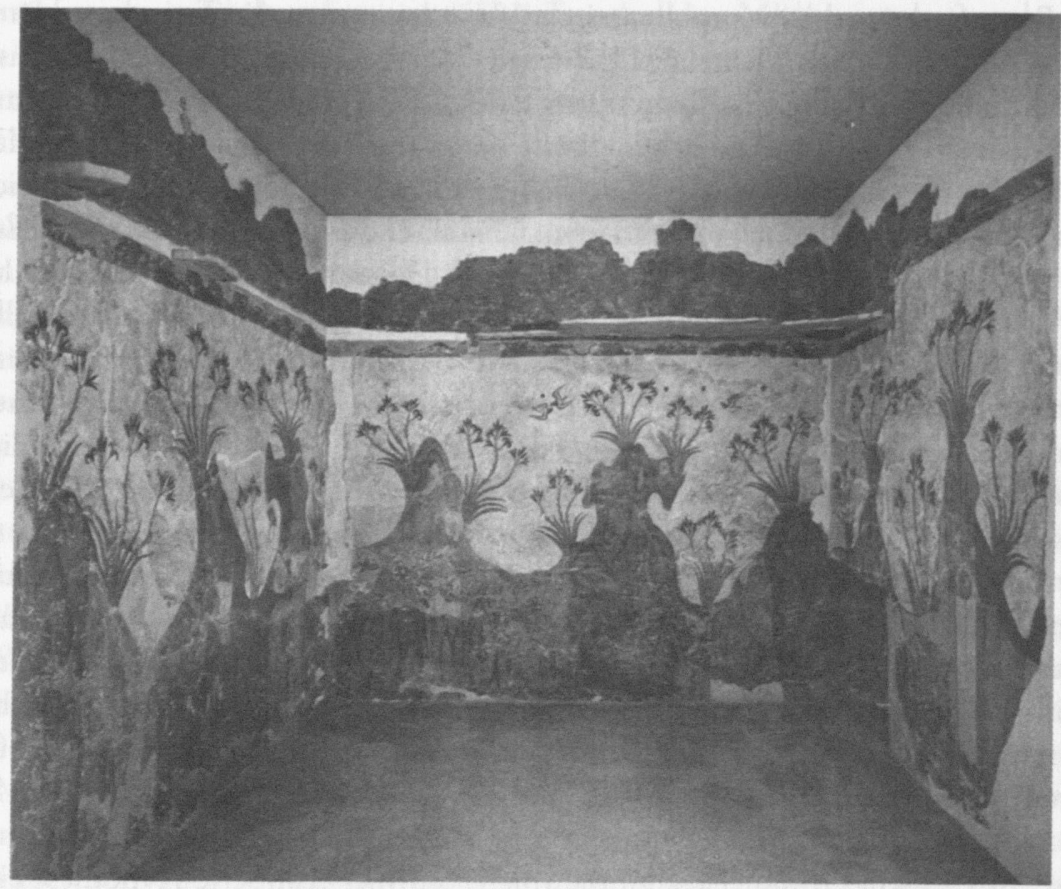

Abb. 6: Akrotiri (Thera): Das, Lilienzimmer' (Raum 2) von Gebäudekomplex Delta.

zeigen unterschiedliche Landschaften: zum einen eine Felsenlandschaft mit Tieren (Süd) und zum anderen eine Gartenlandschaft mit Krokussen und Lilien auf blauem Hintergrund (Nord). Die N-Wand trägt das Bild einer ,knieenden' Frau, die zwischen Krokussen und Lilien dargestellt ist. Ähnliche, vollständig mit Fresken bemalte kleine Räume sind uns aus Akrotiri auf Thera bekannt. ${ }^{75}$ Das ,Lilienzimmer' in Raum 2 von Haus Delta (Abb. 6) zeigt eine dem Ajia Triada-Raum entsprechende Größe (2,20 × 2,60 m, die eine Fläche von 5,72 $\mathrm{m}^{2}$ ergeben). Es weist eine durchgehende Verzierung der Wände mit einer Felsenlandschaft auf, in der Lilien wachsen und Schwalben fliegen. ${ }^{76}$ Auch in diesem Fall war die Beleuchtung des Raumes schlecht und nur durch Öllampen möglich. ${ }^{77}$

Die plausible Interpretation dieser Räume und insbesondere der Inkompatibilität zwischen anspruchsvoller Ausstattung und schlechter visueller Wahrnehmung stellt uns sicherlich vor eine große Herausforderung, die wir nach dem jetzigen Kenntnisstand und ohne schriftliche Quellen schwer bewältigen können.

75 Stürmer, „Naturkulträume“", a. O. (Anm. 72) 71-73.

76 Chr. Doumas, The Wall-Paintings of Thera (1992) 100-107 Abb. 66-76.

77 Stürmer, „Naturkulträume“", a. O. (Anm. 72) 72. 
Die Raumfunktion bleibt problematisch. ${ }^{78}$ Wie kann man die Tatsache erklären, dass die Minoer einen kleinen schlecht beleuchteten Raum, der architektonisch schlicht und unauffällig gestaltet war, mit einem Bildteppich in Freskotechnik ausmalten? Der Widerspruch zwischen Größe und ästhetischer Qualität der gemalten Bilder zum einen und ihrer visuellen Wahrnehmung nur durch künstliches Licht zum anderen, scheint einen rein profanen Charakter dieser Räume auszuschließen. Die vollständige Übermalung der Wände eines kleinen fensterlosen Raumes legt die Vermutung nahe, dass man hier eine Naturwelt in Miniatur schaffen wollte, einen Illusionsraum, den man im Anschluss an M. Foucault als ,Heterotopie ceiner sakralen Landschaft bezeichnen dürfte. ${ }^{79}$ Worin könnte der religiöse oder rituelle Sinn einer solchen ,Heterotopie bestanden haben? Es ist sehr unwahrscheinlich, dass diese Räume als künstlich erschaffene Kulisse für die Durchführung des ekstatischen Rituals fungieren konnten. Obwohl im Mittelpunkt dieses Rituals der Trance-Zustand der Akteure stand, wie bereits angesprochen, war die Ritualaktion offensichtlich nicht introvertiert. Sie muss ihren Sinn nicht in der transzendentalen Erfahrung des Einen, sondern in der Teilhabe und Ergriffenheit der Kultgemeinde erfüllt haben, eine Kultgemeinde, die - wenn auch passiv - diesem Ritual beiwohnte. Die Vorstellung eines orgiastischen Tanzes in einer dunklen, $4 \mathrm{~m}^{2}$ großen Kammer ist in jeder Hinsicht, unnatürlich' und wäre nur im Rahmen eines Mysterienkultes denkbar, an dem nur ein sehr kleiner Personenkreis teilgenommen hätte. Aus diesem Grund erscheint die von V. Stürmer geäußerte Hypothese eines Meditationsraumes für einen minoischen Priester, Schamanen oder eine andere Person nicht ganz abwegig zu sein. ${ }^{80}$ Es ist möglich, dass die Minoer im urbanen Kontext - und zwar in einer dunklen Kammer - einen künstlichen Naturraum, einen abgeschotteten ,Erlebnisraum' erschufen, in dem Priester, Akteure des ekstatischen Rituals oder sogar der Lokalherrscher selbst umgeben von Bildern einer paradiesischen Landschaft, die sie unter dem gedämpften Licht einer Öllampe

78 Eine Deutung dieser Kammern als luxuriöse Schlafräume wegen der vermuteten oder erwiesenen Existenz von Betten kann kaum befriedigend sein, s. Stürmer, „Naturkulträume ““, a.O. (Anm. 72) 73. Das einzige Bett, das tatsächlich in einem dieser Räume entdeckt wurde ('Lilienzimmer' in Gebäudekomplex Delta von Akrotiri) entsprach offensichtlich nicht der ursprünglichen Funktion dieses kleinen Raumes.

79 M. Foucault, „Andere Räume“, in: M. Wentz (Hg.), Stadt-Räume (1991) 68: „Es gibt gleichfalls - und das wohl in jeder Kultur, in jeder Zivilisation - wirkliche Orte, wirksame Orte, die in die Einrichtung der Gesellschaft hineingezeichnet sind, sozusagen Gegenplatzierungen oder Widerlager, tatsächlich realisierte Utopien, in denen die wirklichen Plätze innerhalb der Kultur gleichzeitig repräsentiert, bestritten und gewendet sind, gewissermaBen Orte außerhalb aller Orte, wiewohl sie tatsächlich geortet werden können“.

80 Stürmer, „Naturkulträume“", a.O. (Anm. 72) 73. Aufgrund des häufigen Vorkommens von Wandschränken bzw. -nischen mit Tongefäßen schlägt er ferner eine zusätzliche Funktion als Sakristei vor. 
betrachteten, innehalten konnten. ${ }^{81}$ Der Zweck dieses Meditationsraumes könnte die Aufhebung des Abstands vom dargestellten Prototyp, also der Natur, sowohl in räumlichem als auch in zeitlichem Sinne gewesen sein: räumlich als Überbrückung der geographischen Entfernung von der Wildnis der Berge und zeitlich als Überbrückung der kalten Wintermonate, die den wiederkehrenden Tod der Natur markierten ${ }^{82}$ Diese Hypothese zur Funktionsdeutung der kleinen vollständig ausgemalten Räume gewinnt an Plausibilität, weil sie als einzige mit dem oben vorgeschlagenen Charakter der minoischen religiösen Mentalität konform ist, einer Mentalität, die - gemäß der hier vertretenen Deutung - durch die schöpferische Raumwahrnehmung und die kognitive Erzeugung einer neuen Raumdimension geprägt war.

Der zeitliche Rahmen dieses Prozesses der ,Domestizierung' der naturnahen Riten, ihrer Verpflanzung von der Wildnis der Natur in die geordnete Welt des städtischen Kontextes lässt sich leider nicht mit Sicherheit abstecken. Es gibt keine Anhaltspunkte, dass die sich hier abzeichnende Tendenz, Riten, die ursprünglich außerhalb der Stadt durchgeführt wurden, innerhalb der Grenzen des urbanen Territoriums zu platzieren, eine spätere Entwicklung war, die von einem Wechsel der religiösen Mentalität der Minoer eingeleitet worden wäre. Der einzige Anhaltspunkt für eine solche Vermutung wäre das etwas abrupte Ende der Kultpraxis in den meisten Höhenheiligtümern am Übergang von der Alt- in die Neupalastzeit, das allerdings auch von ganz anderen Faktoren ausgelöst sein könnte. Möglich ist, dass diese beiden Traditionen einer auf die Natur bezogenen Ritualpraxis, die ganz unterschiedlich verortet waren, zeitlich parallel zueinander gelaufen sind.

Eine besondere Erwähnung verdient schließlich in diesem Zusammenhang ein sehr interessanter Einzelfall der bildlichen Raumausstattung. Es handelt sich um ein sehr umfassendes Bildprogramm, in dessen Mittelpunkt wiederum eine Landschaft stand, das jedoch einen ganz anderen rituellen Hintergrund als die oben angesprochenen Szenen hatte. Dieses Bildprogramm erstreckt sich auf mehrere Räume und zwei Stockwerke von Xeste 3, ein Gebäude mit offensichtlich kultischer Funktion in Akrotiri auf Thera. ${ }^{{ }^{3}}$ Inhaltlicher Kern dieses zusammen-

8 I Die punktuelle bzw. graduelle visuelle Wahrnehmung der Wandbilder mit Hilfe des künstlichen Lichts einer Öllampe, diese Inszenierung des Erlebens einer konstruierten Landschaft, hätte zweifellos seine suggestive Wirkung auf den Betrachter gesteigert.

82 Eine überlegenswerte Parallele für die Erschaffung solcher künstlichen Miniaturwelten bieten die seit dem 17. Jh. belegten Anlagen von Gärten in Wasserbecken bei chinesischen Gelehrten, s. Eliade, Das Heilige 134-1 36. Ziel dieser Miniaturlandschaften, die aus Felsen mit Zwergbäumen, Blumen, Miniaturhäusern, Pagoden, Brücken und menschlichen Figuren bestanden, war, einen künstlichen Stimmungsraum zu schaffen, in dem man durch Meditation zur Harmonie mit der Welt gelangen konnte.

83 Doumas, Wall-Paintings of Thera, a. O. (Anm. 76) 127-1 75 Abb. 93-1 37; Marinatos, Ritual, a. O. (Anm. 6) 203-2I I; A.G. Vlachopoulos, „The Wall Paintings from the Xeste 3 Buil- 
hängenden Bildensembles ist das Sammeln von Krokusblüten in einer landschaftlichen Szenerie durch Frauen, die festlich gekleidet sind. Das gesamte Bildprogramm kulminiert in der Szene des Obergeschosses, wo eine Göttin - die größte erhaltene Abbildung einer Gottheit in der ägäischen Kunst -, die von einem Affen und einem Greifen flankiert ist, die Gaben der Krokuspllückerinnen entgegennimmt. Worin besteht der besondere Charakter dieser Bilder in Bezug auf unser Thema, die Sakralisierung der Natur? Sicherlich nicht in der Tatsache, dass der Gottheit Blumen als nicht-blutige Opfer dargebracht werden, eine Praxis, die uns in zahlreichen anderen Kontexten sehr gut dokumentiert ist und kein zwingendes Argument für die Sakralisierung der Landschaft bieten kann. Das ägäische oder minoische Spezifikum besteht hier in der zeitlichen und räumlichen Ausdehnung des Rituals und somit der sakralen Sphäre selbst. Die rituelle Aktion ist nicht auf das Innere bzw. die unmittelbare Umgebung eines Heiligtums bzw. seines Altars beschränkt, sondern beginnt bereits in der Wildnis, mit dem Pflücken oder Auflesen der Gaben der Natur. Dass diese in einem anderen Kontext sicherlich als rein profan betrachtete Aktivität Bestandteil des Rituals war, zeigt nicht nur die große Fläche, die die Bilder des Krokuspflückens in diesem Gebäude einnehmen - und die sicherlich mit ihrer besonderen Bedeutung konform war -, sondern auch die festliche, außeralltägliche Tracht der Krokuspflückerinnen. ${ }^{84}$ Als raumrelevantes Verhalten besitzt die hier geschilderte rituelle Aktion eine ganz besondere Aussagekraft für die Sakralisierung der Landschaft in der ägäischen Bronzezeit.

\section{Die Erschütterung einer vollkommenen Welt?}

Als am Allerheiligen-Tag 1755 Lissabon nach drei kurz aufeinander folgenden heftigen Erdstößen und einer gewaltigen Flutwelle in Trümmern lag, waren nicht nur eine der bedeutendsten europäischen Handelsmetropolen zerstört und, nach schwankenden Schätzungen, 30.000 bis 60.000 Menschen getötet worden. Die schreckliche Naturkatastrophe erschütterte auch die Fundamente des christlichen Glaubens im gesamten Europa und stellte die damals noch junge TheodizeeDebatte auf eine völlig neue Grundlage. ${ }^{85}$ Von einer ähnlichen, wenn nicht noch schrecklicheren Naturkatastrophe wurde auch die minoische Welt heimgesucht:

ding at Akrotiri: Towards an Interpretation of the Iconographic Programme“, in: N. Brodie u. a. (Hgg.), Horizon. A Colloquium on the Prehistory of the Cyclades (2008) 45 I-454.

84 Interessant ist in diesem Fall der Vergleich mit dem Pflücken von Krokussen auf Thera in unserer Zeit, das trotz seines außeralltäglichen Rahmens als eine rein profane Aktivi-

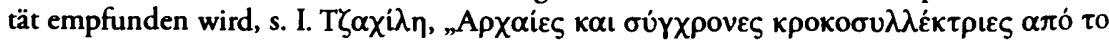

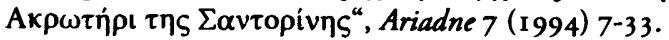

85 W. Breidert (Hg.), Die Erschütterung der vollkommenen Welt. Die Wirkung des Erdbebens von Lissabon im Spiegel europäischer Zeitgenossen (1994). 
dem Vulkanausbruch von Thera. Auch wenn wir nicht unbedingt die Meinung einiger Naturwissenschaftler teilen müssen, wonach es sich dabei um eine der verheerendsten Naturkatastrophen handelte, die die Menschheit erlebte, kann es keinen Zweifel daran geben, dass diese Vulkaneruption dramatische Auswirkungen auf Landschaft und Menschen hatte. Wie stark die minoischen Zentren in Mitleidenschaft gezogen wurden durch Tsunamis, Erdbeben oder Aschenregen, die das Klima in der Region über Jahre oder sogar Jahrzehnte hinweg beeinträchtigt haben müssen, lässt sich immer noch nicht eindeutig sagen. Was in unserem Zusammenhang von besonderem Interesse ist, bezieht sich weniger auf die materiellen Folgen des Vulkanausbruchs, sondern vielmehr auf ihren psychologischen Effekt auf die Gesellschaft, die diese sintflutartige Katastrophe erlebte. Viele Kulturen und Religionen haben gewisse Strategien zur rituellen und sozialen Bewältigung elementarer Gewalten entwickelt. Dasselbe haben sicherlich auch die Minoer im Fall der wiederkehrenden Erdbeben getan. Es ist allerdings sehr zweifelhaft, dass die etablierten Mittel der priesterlichen Elite zur psychologischen Bewältigung von Naturkatastrophen imstande waren, mit dem in ihrer Art und ihrem Ausmaß völlig unfassbaren Vulkanausbruch von Thera zurecht zu kommen. Dieses tragische Ereignis, das sicherlich keinem vorhandenen theologischen Deutungsschema passte, könnte die Fundamente des religiösen Glaubens der Minoer so stark erschüttert haben, so dass ihr harmonisches und rituell überbautes Verhältnis zur Natur auf psychologischer Ebene ,kontaminiert' wurde. Dennoch, trotz der hohen Plausibilität einer sozialen bzw. religiösen Krise, die durch die Thera-Eruption und ihre Folgen ausgelöst wurde, stellt man fest, dass das vermutete kollektive Trauma keine deutlichen Spuren in der archäologischen Überlieferung hinterlassen hat. Es gibt nichts in der minoischen Kunst in der Zeit nach dieser Katastrophe, dass auf ein solches dramatisches Ereignis hinweist. ${ }^{86}$ Einige Jahrzehnte nach dieser Katastrophe bricht die neupalastzeitliche Kultur Kretas zusammen. Im Zuge eines allgemeinen Mentalitätswechsels, dessen Ursachen wir nicht leicht nachvollziehen können, wird die materielle Kultur der Insel von mykenischen Elementen dominiert. Die dinglichen Spuren des Kultes gehören nun einem ganz anderen Kulturhorizont an, den Anfängen der frühgriechischen Geschichte, die uns wesentlich weniger Raum für Spekulationen lässt, als die Zeit, die im Mittelpunkt dieses Beitrags stand.

86 Als einzige mögliche Ausnahme könnte die Beliebtheit der ,Meeresstil'-Keramik auf der Insel unmittelbar nach der Vulkaneruption betrachtet werden, hinter der man eine religiös motivierte Reaktion auf dieses Naturereignis erkennen möchte, s. hierzu J. Driessen - C. MacDonald, The Troubled Island, Minoan Crete before and after the Santorini Eruption. Aegaeum 17 (1997) 98; ferner Müller, Kretische Tongefäße, a. O. (Anm. 18) 322. 
Prof. Dr. Diamantis Panagiotopoulos

Institut für Klassische Archäologie

Universität Heidelberg

Marstallhof 4

D-69117 Heidelberg

Deutschland

diamantis.panagiotopoulos@zaw.uni-heidelberg.de

\section{Abbildungsnachweis:}

Abb. I: Sp. Marinatos, Kreta, Thera und das mykenische Hellas $\left(1973^{2}\right)$ Taf. I.

Abb. 2: M.A.V. Gill - W. Müller - I Pini, Corpus der minoischen und mykenischen Siegel II 8. Iraklion, Archäologisches Museum. Die Siegelabdrücke von Knossos (2002) Nr. 256.

Abb. 3: H. Siebenmorgen (Hg.), Im Labyrinth des Minos. Kreta - die erste europäische Hochkultur (2000) 228, Abb. 186.

Abb. 4: A. Evans, The Falace of Minos at Knossos 2 (1928) 614, Abb. 386.

Abb. 5: J. - E. Sakellarakis, Kreta. Archanes (1991) 79, Abb. 53.

Abb. 6: Sp. Marinatos, Kreta, Thera und das mykenische Hellas (19732) Taf. XXXVII. 